\title{
LEAST SUPERSOLUTION APPROACH TO REGULARIZING FREE BOUNDARY PROBLEMS
}

\author{
DIEGO R. MOREIRA
}

\begin{abstract}
In this paper, we study a free boundary problem obtained as a limit as $\varepsilon \rightarrow 0$ to the following regularizing family of semilinear equations $\Delta u=\beta_{\varepsilon}(u) F(\nabla u)$, where $\beta_{\varepsilon}$ approximates the Dirac delta in the origin and $F$ is a Lipschitz function bounded away from 0 and infinity. The least supersolution approach is used to construct solutions satisfying geometric properties of the level surfaces that are uniform. This allows to prove that the free boundary of the limit has the "right" weak geometry, in the measure theoretical sense. By the construction of some barriers with curvature, the classification of global profiles for the blow-up analysis is carried out and the limit function is proven to be a viscosity and pointwise solution (a.e) to a free boundary problem. Finally, the free boundary is proven to be a $C^{1, \alpha}$ surface around $\mathcal{H}^{n-1}$ a.e. point.
\end{abstract}

\section{INTRODUCTION}

Regularizing methods in free boundary problems are models for a wide spectrum of problems in nature. They are of particular interest in the theory of flame propagation to describe laminar flames as an asymptotic limit for high energy activation. These methods go back to Zeldovich and FrankKamenetski, [ZF], in 1938. However, the rigorous mathematical study was postponed until recently with the pionerring works of Berestycki-CaffarelliNirenberg [BCN] and by Caffarelli-Vazquez [CV].

In the last decade, some attention has been given to the study of limit as $\varepsilon \rightarrow 0$ of solutions to the elliptic equation

$$
\Delta u=\beta_{\varepsilon}(u)
$$

where $\beta_{\varepsilon}(s)=1 / \varepsilon \beta(s / \varepsilon)$ and $\beta$ is a Lipschitz continuous function, with $\beta>0$ in $(0,1), \operatorname{supp}(\beta)=[0,1]$ and $\int \beta=M>0$. It is known from the series of remarkable papers of Luis Caffarelli, Claudia Lederman and Noemi Wolanski, ([CLW1, [CLW2], [LW] ) that under certain geometric conditions about the limit 
function $u_{0}$ and its free boundary, it is a viscosity solution of the following free boundary problem

$$
\left\{\begin{aligned}
\Delta u & =0 & & \text { in } \Omega \backslash\{u>0\} \\
\left(u_{\nu}^{+}\right)^{2}-\left(u_{\nu}^{-}\right)^{2} & =2 M & & \text { on } \Omega \cap \partial\{u>0\}
\end{aligned}\right.
$$

and the free boundary is locally a $C^{1, \alpha}$ surface. These assumptions are necessary if one intends to obtain further regularity results since there are limits which do not satisfy the free boundary condition in the classical sense in any portion of the free boundary ([CLW1], remark 5.1).

Recently, in [CJK], Luis Caffarelli, David Jerison and Carlos Kenig proved some new monotonicity results so that it applies to inhomogeneous equations in which the right-hand side of the equation does not need vanish on the free boundary. The new versions of the monotonicity theorem led to some existence and regularity results to the Prandtl-Batchelor equation. In connection with these results, a uniform Lipschitz estimates for solutions to a family of semilinear equations was proven. These regularizing approximations generalize the type of elliptic equations in (1.1) and they are the object of study of this paper. More concretelly, we study the limit free boundary problem and its regularity theory as $\varepsilon \rightarrow 0$ of the following family of semilinear equations

$$
\Delta u=\beta_{\varepsilon}(u) F(\nabla u)
$$

Here, $F$ is a Lipchitz continuous function bounded away from 0 and infinity. The strategy used in this paper is the following: We use the least supersolution approach to construct solutions $u_{\varepsilon}$, which are more "stable" from the geometric viewpoint. This is done for equations more general than (1.3) and also allows to obtain a limit function with some geometric properties and its free boundary having some "weak" geometry. We then move to study the limit problem. The key part here is the classification of global profiles (2-plane functions) of the blow-up analysis. We remark however that, the typical integration by parts method developed in [CLW1 and extensively used in similar problems does not seem to work for this case. Here, the classification depends upon a delicate construction of barriers with some uniform control on the curvature of their free boundaries as well as the asymptotic behavior of their 
slopes. Finally, the limit of the least supersolutions is proven to be a viscosity and pointwise $\left(\mathcal{H}^{N-1}\right)$ a.e. solution to

$$
\left\{\begin{aligned}
\Delta u & =0 & & \text { in } \Omega \backslash\{u>0\} \\
H_{\nu}\left(u_{\nu}^{+}\right)-H_{\nu}\left(u_{\nu}^{-}\right) & =M & & \text { on } \Omega \cap \partial\{u>0\}
\end{aligned}\right.
$$

with $H_{\nu}(t)=\int_{0}^{t} \frac{s}{F(s \nu)} d s$.

and the free boundary $\Omega \cap \partial\{u>0\}$ to be a $C^{1, \alpha}$ surface around $\mathcal{H}^{n-1}$ a.e. point.

In this case, the free boundary condition

$$
H_{\nu}\left(u_{\nu}^{+}\right)-H_{\nu}\left(u_{\nu}^{-}\right)=M \text { on } F(u)
$$

also depends on the normal direction to the free boundary. This type of free boundary conditions appear as a limit of homogenization problems in periodic media. For homogenization free boundary problems, we refer to [CLM1, CLM2.

\section{Existence, Continuity, Regularity Theory of the Least SUPERSOLUTION}

In this section we will consider the following $\varepsilon$ - regularized equations

$$
\Delta u=F_{\varepsilon}(u, \nabla u) \text { in } \Omega
$$

where $\Omega \subset \mathbb{R}^{N}$ is a Lipschitz domain and $\left\{F_{\varepsilon}\right\}_{\varepsilon>0}$ is under the following structural conditions:

$$
\begin{gathered}
F_{\varepsilon} \in C\left(\mathbb{R} \times \mathbb{R}^{N}\right), \\
0 \leq F_{\varepsilon}(z, p) \leq \frac{A}{\varepsilon} \chi_{\{0<z<\varepsilon\}} \text { in } \mathbb{R} \times \mathbb{R}^{N}, \quad A>0
\end{gathered}
$$

Since our goal is the study of the free boundary of the limit configuration as $\varepsilon \rightarrow 0$, we will be interested to investigate geometric properties of some level sets of $u_{\varepsilon}$. For this reason, we should choose in some sense, more "stable" solutions $u_{\varepsilon}$ to deal with. This was the approach done in [MT], where solutions were chosen to be the minimizers of the corresponding functional associated to 
the $\varepsilon$ - perturbed equations. In this case, due to lack of variational characterization for solutions of $\left(E_{\varepsilon}\right)$, we will consider the least viscosity supersolution of the equation above. This will be accomplished by Perron's method.

Let $\varphi$ be in $C(\partial \Omega)$ and let us define,

$$
\mathcal{S}_{\varphi}^{\varepsilon}=\mathcal{S}_{\varepsilon}:=\left\{w \in C(\bar{\Omega}), w \text { viscosity supersolution of }\left(E_{\varepsilon}\right) ; w \geq \varphi \text { on } \partial \Omega\right\}
$$

Clearly, $\mathcal{S}_{\varepsilon} \neq \emptyset$ since $h_{\varphi} \in \mathcal{S}_{\varepsilon}$, where $h_{\varphi}$ is the harmonic in $\Omega$ such that $h=\varphi$ in $\partial \Omega$. Besides, there is also a natural barrier from below for the functions in set $\mathcal{S}_{\varepsilon}$. Indeed, if for each $\varepsilon>0$, we define

$$
L_{\varepsilon}:=\sup _{(z, p) \in(0, \varepsilon) \times \mathbb{R}^{N}} F_{\varepsilon}(z, p)<+\infty
$$

and let $\Psi_{\varepsilon}$ be the unique solution to

$$
\left\{\begin{aligned}
\Delta \Psi & =L_{\varepsilon} & & \text { in } \Omega \\
\Psi & =\varphi & & \text { on } \partial \Omega,
\end{aligned}\right.
$$

by maximum principle, we have

$$
\mathcal{S}_{\varepsilon}=\left\{w \in C(\bar{\Omega}), w \text { viscosity supersolution of }\left(E_{\varepsilon}\right) ; w \geq \Psi_{\varepsilon} \text { in } \bar{\Omega}\right\}
$$

We define the function

$$
u_{\varepsilon}(x):=\inf _{w \in \mathcal{S}_{\varepsilon}} w(x)
$$

It will be called the least supersolution of the equation $\left(E_{\varepsilon}\right)$. From the discussion above, there exists natural barriers for $u_{\varepsilon}$, namely, $\Psi_{\varepsilon} \leq u_{\varepsilon} \leq h_{\varphi}$ in $\bar{\Omega}$.

Remark 2.1. It worths to notice that, in general, comparison principle for supersolutions and subsolutions of equation $\left(E_{\varepsilon}\right)$ is not available. This way, uniqueness of solutions is not expected to hold.

Remark 2.2. We recall some definitions that are going to be used in next Theorem. If $u: \Omega \rightarrow \mathbb{R}$ is locally bounded, we define

$$
u^{*}(x)=\inf \{v(x) \mid v \in U S C(\Omega) \text { and } v \geq u \text { in } \Omega\}
$$




$$
u_{*}(x)=\sup \{v(x) \mid v \in L S C(\Omega) \text { and } v \leq u \text { in } \Omega\}
$$

Clearly, $u^{*} \in U S C(\Omega), u_{*} \in L S C(\Omega)$ and $u_{*} \leq u \leq u^{*}$. Besides, we have

$$
\begin{aligned}
& u^{*}(x)=\lim _{r \searrow 0} \sup \left\{u(y) \mid y \in \Omega \cap B_{r}(x)\right\} \\
& u_{*}(x)=\liminf _{r \searrow 0}\left\{u(y) \mid y \in \Omega \cap B_{r}(x)\right\}
\end{aligned}
$$

The functions $u^{*}, u_{*}$ are called upper semicontinuous envelope and lower semicontinuous envelope of $u$ respectively.

Theorem 2.3. For each $\varepsilon>0$, the least supersolution of equation $\left(E_{\varepsilon}\right), u_{\varepsilon}$, belongs to $C(\bar{\Omega}) \cap C_{l o c}^{1, \alpha}(\Omega) \cap W_{l o c}^{2, p}(\Omega)$ for any $0<\alpha<1$ and any $1 \leq p<\infty$ and it is a viscosity solution of $\left(E_{\varepsilon}\right)$. Besides, $u_{\varepsilon}$ is a strong solution of $\left(E_{\varepsilon}\right)$ and assume the boundary values $\varphi$ continuously on the boundary, i.e,

$$
\left\{\begin{array}{rlrlrl}
\Delta u_{\varepsilon} & =F_{\varepsilon}\left(u_{\varepsilon}, \nabla u_{\varepsilon}\right) & & \text { a.e. in } & & \Omega \\
u_{\varepsilon} & =\varphi & & \text { on } & \partial \Omega
\end{array}\right.
$$

In particular, $u_{\varepsilon} \in \mathcal{S}_{\varepsilon}$.

Proof. Let us observe first that $u_{\varepsilon}=\left(u_{\varepsilon}\right)^{*}$. It follows from Perron's method developed by Ishii in [I] that $u_{\varepsilon}$ is a a viscosity subsolution and $\left(u_{\varepsilon}\right)_{*}$ is a viscosity supersolution of $\left(E_{\varepsilon}\right)$. Since $\Delta u_{\varepsilon} \geq 0$ in the viscosity sense and $u_{\varepsilon}$ is upper semicontinuous, from the uniqueness of the subharmonic upper semicontinuous representative ([LL], Theorem 9.3), we conclude

$$
u_{\varepsilon}(x)=\lim _{r \rightarrow 0} f_{B_{r}(x)} u_{\varepsilon}(y) d y
$$

Moreover, for any $w \in \mathcal{S}_{\varepsilon}, \Delta w \leq L_{\varepsilon}$ in the viscosity sense. In Particular,

$$
\Delta\left(w-\Psi_{\varepsilon}\right) \leq 0 \text { in } \mathcal{D}^{\prime}(\Omega)
$$

which implies, by the average characterization of superharmonicity,

$$
\Delta\left(u_{\varepsilon}-\Psi_{\varepsilon}\right) \leq 0 \text { in } \mathcal{D}^{\prime}(\Omega)
$$


Again, from superharmonicity theory, there exists a unique superharmonic and lower semicontinuous representative $\omega_{\varepsilon}$ such that $\omega_{\varepsilon}=u_{\varepsilon}-\Psi_{\varepsilon}$ a.e in $\Omega$ and it is given by

$$
\omega_{\varepsilon}(x)=\lim _{r \rightarrow 0} f_{B_{r}(x)}\left[u_{\varepsilon}(y)-\Psi_{\varepsilon}(y)\right] d y=u_{\varepsilon}(x)-\Psi_{\varepsilon}(x)
$$

Where we use (2.6) in the second inequality. In particular, $u_{\varepsilon}$ is lower semicontinuous, and so, $u_{\varepsilon}=\left(u_{\varepsilon}\right)_{*}$ is a continuous viscosity solution of $\left(E_{\varepsilon}\right)$. From the structural conditions of $F_{\varepsilon}$ and the regularity theory developed in [Tr1], there is a universal $0<\gamma<1$ such that, $u_{\varepsilon} \in C_{l o c}^{1, \gamma}(\Omega)$. It also follows from [Tr2] that $u_{\varepsilon}$ is twice differentiable almost everywhere in $\Omega$, with equation $\left(E_{\varepsilon}\right)$ then holding almost everywhere. To finish the proof, observe that if we define, $f_{\varepsilon}(x)=F_{\varepsilon}\left(u_{\varepsilon}(x), \nabla u_{\varepsilon}(x)\right)$, then $f_{\varepsilon} \in C(\Omega) \cap L^{\infty}(\Omega)$ and $\Delta u_{\varepsilon}=f_{\varepsilon}$ in the viscosity sense. From $W^{2, p}$ estimates in ([CC], Theorem 7.1), $u_{\varepsilon} \in W_{l o c}^{2, p}(\Omega)$ for any $1 \leq p<\infty$ and thus $u_{\varepsilon} \in C_{l o c}^{1, \alpha}(\Omega)$ for any $0<\alpha<1$. To finish, let $x_{0} \in \partial \Omega$, and $x_{n} \rightarrow x_{0}$. Since, $\Psi_{\varepsilon}\left(x_{n}\right) \leq u_{\varepsilon}\left(x_{n}\right) \leq h_{\varphi}\left(x_{n}\right)$, letting $n \rightarrow \infty$, we conclude $u\left(x_{0}\right)=\varphi\left(x_{0}\right)$.

Remark 2.4. It follows from the proof of the Theorem (2.3), that under the continuity assumption of $F_{\varepsilon}$ and structural condition 2.2, any continuous viscosity solution of $\left(E_{\varepsilon}\right)$ belongs to $C_{l o c}^{1, \alpha}(\Omega) \cap W_{l o c}^{2, p}(\Omega)$ for any $0<\alpha<1$ and $1 \leq p<\infty$ and satisfies the equation almost everywhere in $\Omega$ and in the distributional sense.

Remark 2.5. The twice differentiability of $u_{\varepsilon}$ in the Theorem above could also be justified by the fact that any function in $W_{l o c}^{2, p}(\Omega)$ with $n<2 p$ is twice diferrentiable almost everywhere. This fact is a consequence of the CalderonZygmund theory. A direct proof can be found in ([CCKS], Appendix C)

To finish this section, we state a result about local uniform Lipschitz regularity. This result is due to Luis Caffarelli.

Theorem 2.6 ([C4], Corollary 2). Let $\left\{v_{\varepsilon}\right\}_{\varepsilon>0}$ be a family of continuous viscosity solutions to $\left(E_{\varepsilon}\right)$ such that $\left\|v_{\varepsilon}\right\|_{L^{\infty}(\Omega)} \leq \mathcal{A}$. Then, if $\Omega^{\prime} \subset \subset \Omega$ there exists a universal contant $C=C\left(\Omega^{\prime}, \mathcal{A}\right)$ such that

$$
\left\|\nabla v_{\varepsilon}\right\|_{L^{\infty}\left(\Omega^{\prime}\right)} \leq C
$$


In particular, the family $\left\{v_{\varepsilon}\right\}_{\varepsilon>0}$ is locally uniformly Lipschitz continuous.

\section{Geometric Properties of the Least Supersolution}

In this section, we prove important geometric properties of the least supersolutions. We will be focused in two properties: Linear growth away from certain level sets and strong nondegeneracy. In general, those properties are not expected to hold for general solutions of the equation $\left(E_{\varepsilon}\right)$. Those properties rely heavily on the special kind of solutions considered, the least supersolutions to $\left(E_{\varepsilon}\right)$. These features will be crucial for the study of the regularity of the free boundary of the limit function later on. As we will see, these geometric facts will imply a rather restrictive geometry of the free boundary.

Some notation is now introduced.

$$
\begin{gathered}
B_{\alpha}^{\star}=B_{\delta_{\varepsilon}}\left(x_{\varepsilon}\right) \text { where } u_{\varepsilon}\left(x_{\varepsilon}\right)=\alpha \text { and } \delta_{\varepsilon}=\frac{1}{2} \operatorname{dist}\left(x_{\varepsilon}, \partial \Omega\right) \\
\Omega_{\alpha}=\left\{x \in \Omega ; 0 \leq u_{\varepsilon}(x) \leq \alpha\right\} \text { and } d_{\alpha}(x)=\operatorname{dist}\left(x, \Omega_{\alpha}\right) \\
\Omega_{\alpha}^{+}=\left\{x \in \Omega ; u_{\varepsilon}(x)>\alpha\right\} \\
\Omega^{\prime} \subset \subset \Omega \text { and } \Delta=\operatorname{dist}\left(\Omega^{\prime}, \mathbb{R}^{N} \backslash \Omega\right)
\end{gathered}
$$

Theorem 3.1 (Linear growth away from level set $\varepsilon$ ). There exists a universal constant $C_{3}>0$ such that if $x_{0} \in B_{\varepsilon}^{\star} \cap \Omega_{\varepsilon}^{+}$

$$
u_{\varepsilon}\left(x_{0}\right) \geq C_{3} d_{\varepsilon}\left(x_{0}\right)
$$

Proof. Let us prove by contradiction. If this is not the case, for $\varepsilon>0$ small enough, there exists $y_{\varepsilon} \in B_{\varepsilon}^{\star} \cap \Omega_{\varepsilon}^{+}$such that $u_{\varepsilon}\left(y_{\varepsilon}\right)<<d_{\varepsilon}\left(y_{\varepsilon}\right)=d_{\varepsilon}$. The idea now, is to construct an admissible supersolution (in $\mathcal{S}_{\varepsilon}$ ) strictly below $u_{\varepsilon}$ in some point providing a contradiction. Since, $y_{\varepsilon} \in B_{\varepsilon}^{\star} \cap \Omega_{\varepsilon}^{+}$, we have $B_{d_{\varepsilon}}\left(y_{\varepsilon}\right) \subset \Omega_{\varepsilon}^{+}$and thus

$$
\Delta u_{\varepsilon}=0 \text { in } B_{d_{\varepsilon}}\left(y_{\varepsilon}\right)
$$

By Harnack inequality, there exists a universal constant $C>0$ such that

$$
u_{\varepsilon} \leq C u_{\varepsilon}\left(y_{\varepsilon}\right) \text { in } B_{d_{\varepsilon} / 2}\left(y_{\varepsilon}\right)
$$


Now, consider the following function:

$$
\left\{\begin{array}{rll}
\Delta v_{\varepsilon}=0 & \text { in } \mathcal{R}=B_{d_{\varepsilon} / 2}\left(y_{\varepsilon}\right) \backslash \overline{B_{d_{\varepsilon} / 4}\left(y_{\varepsilon}\right)} \\
v_{\varepsilon}=0 & \text { on } \partial B_{d_{\varepsilon} / 4}\left(y_{\varepsilon}\right) \\
v_{\varepsilon}=1 & \text { on } \partial B_{d_{\varepsilon} / 2}\left(y_{\varepsilon}\right)
\end{array}\right.
$$

and define,

$$
\overline{w_{\varepsilon}}=\left\{\begin{aligned}
0, & \text { in } \overline{B_{d_{\varepsilon / 4}}\left(y_{\varepsilon}\right)} \\
\min \left\{u_{\varepsilon}, d_{\varepsilon} v_{\varepsilon}\right\} & \text { in } \mathcal{R}=B_{d_{\varepsilon} / 2}\left(y_{\varepsilon}\right) \backslash \overline{B_{d_{\varepsilon} / 4}\left(y_{\varepsilon}\right)} \\
u_{\varepsilon} & \text { in } \Omega \backslash \overline{B_{d_{\varepsilon} / 2}\left(y_{\varepsilon}\right)}
\end{aligned}\right.
$$

Since $C>0$ is a universal constant (that appears in the Harnack inequality) and $u_{\varepsilon}\left(x_{0}\right)<<d_{\varepsilon}$, we can assume for $\varepsilon$ small enough that, $C u_{\varepsilon}\left(x_{0}\right)<d_{\varepsilon}$, and thus, $\overline{w_{\varepsilon}}$ is continuous along $\partial B_{d_{\varepsilon} / 4}\left(y_{\varepsilon}\right)$. It is easy to check that, $\overline{w_{\varepsilon}}$ is a supersolution ([C], Proposition 2.8, for example) and so $\overline{w_{\varepsilon}} \in \mathcal{S}_{\varepsilon}$, providing a contradiction since $\overline{w_{\varepsilon}}\left(x_{0}\right)=0<u_{\varepsilon}\left(x_{0}\right)$. This finishes the proof of the Theorem.

In what follows, we will assume that the family $\left\{u_{\varepsilon}\right\}_{\varepsilon>0}$ of least supersolutions of the equation $\left(E_{\varepsilon}\right)$ is uniformly bounded, i .e,

$$
\left\|u_{\varepsilon}\right\|_{L^{\infty}(\Omega)} \leq \mathcal{A}
$$

Corollary 3.2. There exists a universal constant $C=C\left(\Omega^{\prime}, \mathcal{A}\right)$ such that

$$
x \in \Omega^{\prime} \cap \Omega_{\varepsilon}^{+}, d_{\varepsilon}(x) \leq \frac{\Delta}{4} \Longrightarrow C_{3} d_{\varepsilon}(x) \leq u_{\varepsilon}(x) \leq C d_{\varepsilon}(x)+\varepsilon
$$

Proof. The first inequality follows from the Theorem (3.1) just by observing that if $d_{\varepsilon}(x)<\frac{\Delta}{3}$, then $x \in B_{\varepsilon}^{\star}$. Indeed, let $x_{\varepsilon} \in \partial \Omega_{\varepsilon}^{+}$with $d_{\varepsilon}(x)=\left|x-x_{\varepsilon}\right|$, then

$$
2\left|x-x_{\varepsilon}\right|=2 d_{\varepsilon}(x)<\operatorname{dist}(x, \partial \Omega)-d_{\varepsilon}(x) \leq \operatorname{dist}\left(x_{\varepsilon}, \partial \Omega\right)=2 \delta_{\varepsilon}(x)
$$

The other inequality follows from uniform Lipschitz continuity, Theorem (2.6). 
We turn our attention to a strong nondegeneracy result for the least supersolutions. Below, we state the Strong Nondegeneracy Lemma. The proof can be found in ([CS], Theorem 1.19) for the Laplacian or in ([MT], Lemma 3.3) for a general divergence operator with Holder coefficients.

Lemma 3.3. (Strong Nondegeneracy Lemma) Assume that $v \geq 0$ is a Lipschitz and harmonic in $\Omega \cap B_{R}(\xi)$, such that

(1) $v \equiv \delta$ on $\partial \Omega \cap B_{R}(\xi), \xi \in \partial \Omega$

(2) $v\left(x_{0}\right) \geq C \delta>0, C>>1$ with $x_{0} \in B_{R / 2}(\xi)$

(3) $v(x) \geq D \cdot \operatorname{dist}(x, \partial \Omega)$ in $\{v \geq C \delta\} \cap B_{R / 2}(\xi)$

Then, there exists a universal constant $M=M(C, D$, Lip $(v))$ such that:

$$
\sup _{B_{r}\left(x_{0}\right)} v \geq M r \text { for } 0<r \leq \frac{R}{4}
$$

Theorem 3.4 (Strong Nondegeneracy). Given $C_{4}>1$ there exists $C=$ $C\left(\Omega^{\prime}, C_{3}, C_{4}, \mathcal{A}\right)$ such that

$$
\sup _{B_{\rho}\left(x_{0}\right)} u_{\varepsilon} \geq C \rho \quad \text { for } \rho \leq \frac{\Delta}{12}
$$

for

$$
x_{0} \in \Omega^{\prime} \cap\left\{u_{\varepsilon} \geq C_{4} \varepsilon\right\}, d_{\varepsilon}\left(x_{0}\right) \leq \frac{\Delta}{6}
$$

Proof. Let $x_{0}$ be in the conditions above. If $d_{\varepsilon}=d_{\varepsilon}\left(x_{0}\right)=\left|x_{0}-x_{\varepsilon}\right|$ and $\delta_{\varepsilon}=\operatorname{dist}\left(x_{\varepsilon}, \partial \Omega\right)$, we have:

This way,

$$
\frac{2 \Delta}{3}=\Delta-\frac{\Delta}{3}<\operatorname{dist}\left(x_{0}, \partial \Omega\right)-d_{\varepsilon}\left(x_{0}\right) \leq \delta_{\varepsilon}
$$

$$
x_{0} \in \frac{1}{2} \overline{B_{\frac{\Delta}{3}}\left(x_{\varepsilon}\right)} \subset B_{\frac{\Delta}{3}}\left(x_{\varepsilon}\right) \subset \frac{1}{2} B_{\delta_{\varepsilon}}\left(x_{\varepsilon}\right)=B_{\varepsilon}^{\star}
$$

Besides,

$$
\bigcup B_{\frac{\Delta}{3}}\left(x_{\varepsilon}\right) \subset \mathcal{N}_{\frac{\Delta}{2}}\left(\Omega^{\prime}\right) \subset \subset \Omega
$$

The inclusion (3.4) and Theorem (3.1) say that the previous Lemma can be used with $B_{\frac{\Delta}{3}}\left(x_{\varepsilon}\right)$ in place of $B_{R}(\xi)$ and the last inclusion 3.5 says that we 
can take uniform Lipschitz for all the appearing balls there. This concludes the Theorem.

\section{The Limit of the LEAST SUPERSOlutions}

This section will be devoted to establish the first results about the limit function and the weak geometry of its free boundary. Before, we introduce the following notation for a continuous function $v: \Omega \rightarrow \mathbb{R}$

$$
\begin{gathered}
\Omega^{+}(v)=\{x \in \Omega \mid v(x)>0\} ; \Omega^{-}(v)=\left(\Omega \backslash \Omega^{+}(v)\right)^{\circ} \\
F(v)=\partial\{x \in \Omega \mid v(x)>0\} \cap \Omega=\partial \Omega^{+} \cap \Omega
\end{gathered}
$$

The set $F(v)$ is called the free boundary of $v$. Again, in what follows, we assume $\Omega^{\prime} \subset \subset \Omega$.

Theorem 4.1. [Properties of the limit of the least supersolutions]

Let $\left\{u_{\varepsilon}\right\}_{\varepsilon>0}$ the family of least supersolutions of $\left(E_{\varepsilon}\right)$. Assume,

$$
\left\|u_{\varepsilon}\right\|_{L^{\infty}(\Omega)} \leq \mathcal{A}
$$

Then for every sequence $\varepsilon_{k} \rightarrow 0$ there exists a subsequence $\varepsilon_{k}^{\prime} \rightarrow 0$ such that

a) $u_{\varepsilon_{k}^{\prime}} \rightarrow u_{0} \in C_{l o c}^{0,1}(\Omega)$ uniformly on compact subsets of $\Omega$.

b) (Regularity) $u_{0} \in C_{l o c}^{0,1}(\Omega), \Delta u_{0} \geq 0$ in $\mathcal{D}^{\prime}(\Omega)$ and

$$
\Delta u_{0}=0 \text { in } \Omega^{+}\left(u_{0}\right) \text { and in } \Omega^{-}\left(u_{0}\right)
$$

c) (Linear growth away of the free boundary) Let $C_{3}>0$ be the constant given by Theorem (3.1), then

$$
\begin{gathered}
u_{0}^{+}\left(x_{0}\right) \geq C_{3} \operatorname{dist}\left(x_{0},\left\{u_{0} \leq 0\right\}\right) \\
\text { if } x_{0} \in \Omega^{\prime}, \operatorname{dist}\left(x_{0},\left\{u_{0} \leq 0\right\}\right) \leq \frac{\Delta}{4}
\end{gathered}
$$


d) (Strong nondegeneracy) There exists a constant $C=C\left(\Omega^{\prime}, \mathcal{A}\right)$ such that:

$$
\sup _{B_{\rho}\left(x_{0}\right)} u_{0} \geq C \rho \text { for } \rho \leq \frac{\Delta}{12}
$$

provided

$$
x_{0} \in \Omega^{\prime} \cap\left(\Omega_{0} \cup F\left(u_{0}\right)\right) \text { with } \operatorname{dist}\left(x_{0},\left\{u_{0} \leq 0\right\}\right) \leq \frac{\Delta}{6}
$$

e) (Nondegeneracy) There exists a constant $\underline{C}=\underline{C}\left(\Omega^{\prime}, \mathcal{A}\right)$ and $\bar{C}=\bar{C}\left(\Omega^{\prime}, \mathcal{A}\right)$ such that:

$$
\underline{C} \leq \frac{1}{\rho} f_{\partial B_{\rho}\left(x_{0}\right)} u_{0}^{+}(y) d \mathcal{H}^{N-1}(y) \leq \bar{C} \quad \text { for } \rho \leq \frac{\Delta}{12}
$$

whenever

$$
x_{0} \in \Omega^{\prime} \cap F\left(u_{0}\right) \text { with } \operatorname{dist}\left(x_{0},\left\{u_{0} \leq 0\right\}\right) \leq \frac{\Delta}{6}
$$

Proof. a) follows imediately from Theorem (2.6) and Ascoli-Arzela Theorem. In $b$ ), the subharmonicity of $u_{0}$ is a straightforward consequence of the average characterization of subharmonic functions and the uniform convergence. To prove $\Delta u_{0}=0$ in $\Omega^{+}\left(u_{0}\right)$, let $B$ be a ball $B \subset \subset \Omega^{+}\left(u_{0}\right)$. There exists $c>0$ such that $u_{0} \geq c$ in $\bar{B}$. From the uniform convergence, if $\varepsilon_{k}^{\prime}$ is taken small enough, $u_{\varepsilon_{k}^{\prime}} \geq \frac{c}{2} \geq \varepsilon_{k}^{\prime}$ in $\bar{B}$. So, $\Delta u_{\varepsilon_{k}^{\prime}}=0$ in $B$ and thus, $\Delta u_{0}=0$ in $B$. Analogously, we conclude, $\Delta u_{0}=0$ in $\left\{u_{0}<0\right\}$. This implies, $\Delta u_{0} \leq 0$ in $\Omega^{-}\left(u_{0}\right)$. From the global subharmonicity of $u_{0}$ we conclude $b$ ). To prove $\left.c\right)$, we can assume $x_{0} \in \Omega^{+}\left(u_{0}\right) \cap \Omega^{\prime}$. For $\varepsilon_{k}^{\prime}>0$ small enough, we have $x_{0} \in \Omega_{\varepsilon_{k}^{\prime}}^{+}$ and $d_{\varepsilon_{k}^{\prime}}\left(x_{0}\right) \leq \frac{\Delta}{4}$. By Corollary $(3.2)$, there exists $y_{k} \in \Omega_{\varepsilon_{k}^{\prime}}, d_{\varepsilon_{k}^{\prime}}\left(x_{0}\right)=\left|x_{0}-y_{k}\right|$ such that:

$$
u_{\varepsilon_{k}^{\prime}}\left(x_{0}\right) \geq C_{3} d_{\varepsilon_{k}^{\prime}}\left(x_{0}\right)=C_{3}\left|x_{0}-y_{k}\right|
$$

Since $\left\{y_{k}\right\}_{k \geq 1}$ is bounded, we can assume, $y_{k} \rightarrow y_{0}, y_{0} \in \Omega \backslash \Omega^{+}\left(u_{0}\right)$ and thus,

$$
u_{0}\left(x_{0}\right) \geq C_{3}\left|x_{0}-y_{0}\right| \geq C_{3} \operatorname{dist}\left(x_{0},\left\{u_{0} \leq 0\right\}\right)
$$


For $d)$, let us study two cases: $(i) x_{0} \in \Omega^{+}\left(u_{0}\right)$ and $(i i) x_{0} \in F\left(u_{0}\right)$. In case (i) again, for $\varepsilon_{k}^{\prime}>0$ small enough, we have $x_{0} \in \Omega_{C_{4} \varepsilon_{k}^{\prime}}^{+}$and $d_{\varepsilon_{k}^{\prime}}\left(x_{0}\right) \leq \frac{\Delta}{6}$. By Theorem (3.4),

$$
\sup _{B_{\rho}\left(x_{0}\right)} u_{\varepsilon_{k}^{\prime}} \geq C \rho
$$

Since $u_{\varepsilon_{k}^{\prime}} \rightarrow u_{0}$ uniformly in compact subsets, passing the limit in the previous expression, the result is proven. Now, to prove (ii) let us observe that we can find $x_{1} \in \Omega^{+}\left(u_{0}\right) \cap B_{\rho / 4}\left(x_{0}\right)$. Setting, $K=\mathcal{N}_{\frac{\Delta}{8}}\left(\Omega^{\prime}\right)$ and applying $(i)$ to $K$, we conclude

$$
\sup _{B_{\rho}\left(x_{0}\right)} u_{0} \geq \sup _{B_{\rho / 4}\left(x_{1}\right)} u_{0} \geq \frac{C \rho}{4}
$$

e) follows from $b$ ) and $d$ ). Indeed, if we define $K=\mathcal{N}_{\frac{\Delta}{2}}\left(\Omega^{\prime}\right)$, then $\overline{B_{\rho}\left(x_{0}\right)} \subset K$ and by Lipschitz continuity,

$$
u_{0}^{+} \leq\left(\frac{\operatorname{Lip}\left(u_{0} \mid K\right)}{12}\right) \rho \text { in } B_{\rho}\left(x_{0}\right)
$$

yielding,

$$
\frac{1}{\rho} \int_{\partial B_{\rho}\left(x_{0}\right)} u_{0}^{+} d \mathcal{H}^{N-1} \leq \bar{C}
$$

To prove the other inequality, let us consider $x_{0}$ in the conditions described in $e$ ). This way, by $d$ ), there exists $x_{1} \in \overline{B_{\rho / 2}\left(x_{0}\right)}$ such that $u_{0}\left(x_{1}\right) \geq \frac{C \rho}{4}$. By, Lipschitz continuity, if $\tau \leq \frac{1}{3}$, since $B_{\rho \tau}\left(x_{1}\right) \subset \subset B_{\rho}\left(x_{0}\right) \subset K$

$$
u_{0} \geq\left(\frac{C}{4}-\operatorname{Lip}\left(u_{0} \mid K\right) \tau\right) \rho \text { in } B_{\rho \tau}\left(x_{1}\right)
$$

Taking $\tau$ small enough, $u_{0} \geq \frac{C \rho}{8}>0$ in $B_{\rho \tau}\left(x_{1}\right)$ and thus,

$$
f_{B_{\rho}\left(x_{0}\right)} u_{0}^{+} d x \geq \tau^{N-1} f_{B_{\rho \tau}\left(x_{1}\right)} u_{0}^{+} d x \geq \frac{\tau^{N} C}{8} \rho
$$

By now, we have proven $e$ ) for the volume average, i.e, there exist a constant $C_{1}=C_{1}\left(\Omega^{\prime}, \mathcal{A}\right)>0$ such that, whenever, $x_{0}$ is in the conditions of $(5)$, we have:

$$
\frac{1}{\rho} \int_{B_{\rho}\left(x_{0}\right)} u_{0}^{+} d x \geq C_{1}
$$


From the fact that $u_{0}^{+} \geq 0$ is locally Lipschitz constinuous and harmonic in $\left\{u_{0}^{+}>0\right\}$, the same conclusion holds for the area average as in the statement of $e$ ). Indeed, suppose by contradiction, that this is not the case. Then, we can find a sequence $\left\{x_{n}\right\}_{n \geq 1} \subset F\left(u_{0}\right) \cap \Omega^{\prime}$ with $\operatorname{dist}\left(x_{n},\left\{u_{0} \leq 0\right\}\right) \leq \frac{\Delta}{6}$, such that

$$
f_{\partial B_{\rho_{n}}\left(x_{n}\right)} u_{0}^{+} d \mathcal{H}^{N-1} \leq \frac{1}{n} \rho_{n} \text { with } \rho_{n} \rightarrow 0 .
$$

Considering the rescaling functions, $v_{n}(x):=\frac{1}{\rho_{n}} u_{0}^{+}\left(x_{n}+\rho_{n} x\right)$, it follows from remark 4.2), there exists a subsequence, that we still denote by $v_{n}$, such that $v_{n} \rightarrow V$ uniformly in compact sets of $\mathbb{R}^{N}, V \geq 0, V$ Lipschitz continuous an harmonic in $\{V>0\}$. Now, rewriting, (4.2), in terms of $v_{n}$ we find,

$$
f_{\partial B_{1}(0)} v_{n} d \mathcal{H}^{N-1} \leq \frac{1}{n} \rho_{n}
$$

Since, $u_{0}^{+}$is globally subharmonic, we have

$$
0 \leq f_{B_{1}(0)} u_{0}^{+} d x \leq f_{\partial B_{1}(0)} u_{0}^{+} d \mathcal{H}^{N-1}=0
$$

which implies that $u_{0}^{+} \equiv 0$ in $B_{1}(0)$. On the other hand, we have proven that

$$
f_{B_{1}(0)} v_{n} d x \geq C_{1}>0
$$

Letting $n \rightarrow \infty$, we get $f_{B_{1}(0)} u_{0}^{+} d x \geq C_{1}>0$, a contradiction. This finishes the proof of the Theorem.

Remark 4.2. Let us observe that the Lipschitz constant is invariant under the rescaling $v_{n}(x):=\frac{1}{\rho_{n}} u_{0}^{+}\left(x_{n}+\rho_{n} x\right)$. Moreover, $v_{n}(0)=0$ for all $n \geq 1$. So, we can obtain a function $V$ described as in the proof of theorem 4.1) by AscoliArzela Theorem. Since $v_{n}^{\prime} s$ are harmonic whenever positive, by the uniform convegernce, the same holds for $V$.

Now, we establish some properties of the free boundary of $u_{0}, F\left(u_{0}\right)$. Before, we need the following definition 
Definition 4.3. Let $v: \Omega \rightarrow \mathbb{R}$ be a continuous function. A unit vector $\nu \in \mathbb{R}^{N}$ is said to be the inward unit normal in the measure theoretic sense to the free boundary $F(v)$ at a point $x_{0} \in F(v)$ if

$$
\lim _{\rho \rightarrow 0} \frac{1}{\rho^{N}} \int_{B_{\rho}\left(x_{0}\right)}\left|\chi_{\{v>0\}}-\chi_{H_{\nu}^{+}\left(x_{0}\right)}\right| d x=0
$$

where $H_{\nu}^{+}\left(x_{0}\right)=\left\{x \in \mathbb{R}^{N} \mid\left\langle x-x_{0}, \nu\right\rangle>0\right\}$. If $A$ is a set of locally finite perimeter, then for every point in the reduced boundary, $\partial_{\text {red }} A$, the inward unit normal is defined. The details can be found in ([EG], section 5.7).

Theorem 4.4. (Properties of the free boundary $F\left(u_{0}\right)$ )

Let $u_{0}$ be the functions given by Theorem (4.1). Then,

a) $\mathcal{H}^{N-1}\left(\Omega^{\prime} \cap \partial\left\{u_{0}>0\right\}\right)<\infty$

b) There exist borelian functions $q_{u_{0}}^{+}$and $q_{u_{0}}^{-}$defined on $F\left(u_{0}\right)$ such that

$$
\begin{aligned}
\Delta u_{0}^{+} & =q_{u_{0}}^{+} \mathcal{H}^{N-1}\left\lfloor\partial\left\{u_{0}>0\right\}\right. \\
\Delta u_{0}^{-} & =q_{u_{0}}^{-} \mathcal{H}^{N-1}\left\lfloor\partial\left\{u_{0}>0\right\}\right.
\end{aligned}
$$

c) There exists universal constants $\underline{C}>0, \bar{C}>0$ and $\rho_{0}>0$ depending on $\Omega^{\prime}, \mathcal{A}$ such that

$$
\underline{C} \rho^{N-1} \leq \mathcal{H}^{N-1}\left(B_{\rho}\left(x_{0}\right) \cap \partial\left\{u_{0}>0\right\}\right) \leq \bar{C} \rho^{N-1}
$$

for every $x_{0} \in \Omega^{\prime} \cap\left\{u_{0}>0\right\}, 0<\rho<\rho_{0}$

d) $0<\underline{C} \leq q_{u_{0}}^{+} \leq \bar{C}$ and $0 \leq q_{u_{0}}^{-} \leq \bar{C}$ in $\Omega^{\prime}\left\{u_{0}>0\right\}$. In addition, $q_{u_{0}}^{-}=0$ in $\partial\left\{u_{0}>0\right\} \backslash\left\{u_{0}<0\right\}$.

e) $u_{0}$ has the following asymptotic development at $\mathcal{H}^{N-1}$-almost every point $x_{0}$ in $F\left(u_{0}\right)_{\text {red }}$

$$
u_{0}(x)=q_{u_{0}}^{+}\left(x_{0}\right)\left\langle x-x_{0}, \nu\right\rangle^{+}-q_{u_{0}}^{-}\left(x_{0}\right)\left\langle x-x_{0}, \nu\right\rangle^{-}+o\left(\left|x-x_{0}\right|\right)
$$

f) There exists a constant $\tau=\tau\left(\Omega^{\prime}, \mathcal{A}\right)>0$ such that

$$
\mathcal{H}^{N-1}\left(F\left(u_{0}\right)_{\text {red }} \cap B_{\rho}\left(x_{0}\right)\right) \geq \tau^{\star} \rho^{N-1}
$$


for any $x_{0} \in F\left(u_{0}\right) \cap \Omega^{\prime}$. In Particular, we have

$$
\mathcal{H}^{N-1}\left(F\left(u_{0}\right) \backslash F\left(u_{0}\right)_{\text {red }}\right)=0
$$

Proof. It follows from Theorem 4.1 that all the assumptions of the AltCaffarelli Theory developed in [AC] section 4 are satisfied. This way, it is proven there that $a), b), c), d$ ) and $e$ ) holds. A brief overview can be found in [LW], Theorem (3.2). We observe however, that because of the lack of variational characterization for solutions $u_{\varepsilon}$ (and therefore for $u_{0}$ ), we are unable to obtain a positive uniform density from below of the positive phase, like Lemma (3.7) in $\mathrm{AC}$. Then, the $\mathcal{H}^{N-1}$ measure totality in 4.4) of the reduced free boundary, $F\left(u_{0}\right)_{\text {red }}$, does not follow from Alt-Caffarelli theory in [AC]. Instead, a sublte construction like one developed in [C3] is necessary. This way, let us concentrate in proving $f$ ). By rescaling, i.e, considering the function

$$
\left(u_{0}\right)_{\rho}(x)=\frac{1}{\rho} u_{0}\left(\rho\left(x-x_{0}\right)\right)
$$

it is enough to prove the case where $\rho=1$ and $x_{0}=0$. For $0<\sigma<1 / 4$, let us define the following auxiliary function $v_{\sigma}$

$$
\left\{\begin{aligned}
\Delta v_{\sigma} & =-\frac{1}{\left|B_{\sigma}(0)\right|} \chi_{B_{\sigma}(0)} & & \text { in } B_{1}(0) \\
v_{\sigma} & =0 & & \text { on } \partial B_{1}(0)
\end{aligned}\right.
$$

In fact, if $G(x, y)$ denotes the Green function of the unit ball, we have

$$
v_{\sigma}(x)=-\int_{B_{\sigma}(0)} G(x, y) d y
$$

By maximum principle, $v_{\sigma} \geq 0$. It follows from Litmann-WeinbergerStampacchia Theorem, ([LWS], Theorem 7.1), that $v_{\sigma} \leq \bar{C} \sigma^{2-N}$ outside $B_{2 \sigma}(0)$ $\left(\bar{C}>0\right.$ universal constant) and $\partial_{\nu} v_{\sigma} \sim C>0$ (here, $C$ is also a universal constant) along $\partial B_{1}(0)$, where $\nu$ is the unit outwards normal vector to $\partial B_{1}(0)$. Now, by Harnack inequality, ([GT], Theorems 8.17 and 8.18), for any $q>\frac{N}{2}$,

$$
\sup _{B_{2 \sigma}(0)} v_{\sigma} \leq C^{\star}\left\{\inf _{B_{2 \sigma}(0)} v_{\sigma}+\sigma^{2-\frac{2 N}{q}}|| \frac{1}{\left|B_{\sigma}(0)\right|} \chi_{B_{\sigma}(0)} \|_{L^{q}\left(B_{2 \sigma}(0)\right)}\right\}
$$


where $C^{\star}=C^{\star}(N, q)$. Since $\inf _{B_{\sigma}(0)} v_{\sigma} \leq \bar{C} \sigma^{2-N}$, we finally obtain that

$$
v_{\sigma} \leq C \sigma^{2-N} \text { in } B_{1}(0), \text { where } C=C(N, \sigma)
$$

Since, $u_{\varepsilon_{k}}, v_{\sigma} \in C^{1, \alpha}\left(\overline{B_{1}(0)}\right)$ for any $0<\alpha<1$, we can apply the second Green's formula, obtaining

$$
\begin{gathered}
\int_{\Omega^{+}\left(u_{0}\right) \cap B_{1}(0)}\left(v_{\sigma} \Delta u_{\varepsilon_{k}}-u_{\varepsilon_{k}} \Delta v_{\sigma}\right) d x= \\
=\int_{B_{1}(0) \cap F\left(u_{0}\right)_{\text {red }}}\left(v_{\sigma} \partial_{\nu} u_{\varepsilon_{k}}-u_{\varepsilon_{k}} \partial_{\nu} v_{\sigma}\right) d \mathcal{H}^{N-1}-\int_{\partial B_{1}(0) \cap \Omega^{+}\left(u_{0}\right)} u_{\varepsilon_{k}} \partial_{\nu} v_{\sigma} d \mathcal{H}^{N-1}
\end{gathered}
$$

From the uniform Lipschitz continuity of $u_{\varepsilon_{k}}$ in $\overline{B_{1}(0)}$ and 4.6 ,

$$
\left|\int_{B_{1}(0) \cap F\left(u_{0}\right)_{\text {red }}} v_{\sigma} \partial_{\nu} u_{\varepsilon_{k}} d \mathcal{H}^{N-1}\right| \leq C \sigma^{2-N} \mathcal{H}^{N-1}\left(F\left(u_{0}\right)_{\text {red }} \cap B_{1}(0)\right)
$$

Moreover, as $\varepsilon_{k} \rightarrow 0$,

$$
\begin{gathered}
\int_{B_{1}(0) \cap F\left(u_{0}\right)_{\text {red }}} u_{\varepsilon_{k}} \partial_{\nu} v_{\sigma} d \mathcal{H}^{N-1} \rightarrow 0 \\
\int_{\partial B_{1}(0) \cap \Omega^{+}\left(u_{0}\right)} u_{\varepsilon_{k}} \partial_{\nu} v_{\sigma} d \mathcal{H}^{N-1} \rightarrow \int_{\partial B_{1}(0)} u_{0}^{+} \partial_{\nu} v_{\sigma} d \mathcal{H}^{N-1} \\
-\int_{\Omega^{+}\left(u_{0}\right) \cap B_{1}(0)} u_{\varepsilon_{k}} \Delta v_{\sigma} d x=\frac{1}{\left|B_{\sigma}(0)\right|} \int_{\Omega^{+}\left(u_{0}\right) \cap B_{\sigma}(0)} u_{\varepsilon_{k}} d x \rightarrow f_{B_{\sigma}(0)} u_{0}^{+} d x
\end{gathered}
$$

Since $v_{\sigma} \Delta u_{\varepsilon_{k}} \geq 0$, from 4.7), we deduce

$$
f_{B_{\sigma}(0)} u_{0}^{+} d x+\int_{\partial B_{1}(0)} u_{0}^{+} \partial_{\nu} v_{\sigma} d \mathcal{H}^{N-1} \leq C \sigma^{2-N} \mathcal{H}^{N-1}\left(F\left(u_{0}\right)_{\text {red }} \cap B_{1}(0)\right)
$$

By Theorem (4.1)-e),

$$
\int_{\partial B_{1}(0) \cap \Omega^{+}\left(u_{0}\right)} u_{0}^{+} \partial_{\nu} v_{\sigma} d \mathcal{H}^{N-1} \geq C_{1}>0
$$


In Particular, again by nondegeneracy (4.1), the relation (4.8) implies

$$
C^{\star} \sigma \leq f_{B_{\sigma}(0)} u_{0}^{+} d x \leq C \sigma^{2-N} \mathcal{H}^{N-1}\left(F\left(u_{0}\right)_{\text {red }} \cap B_{1}(0)\right)
$$

To conclude, since there exist $\underline{C}, \bar{C}$ depending on $\Omega^{\prime}$ and $\mathcal{A}$, such that

$$
\underline{C} \leq \frac{1}{\sigma} f_{B_{\sigma}(0)} u_{0}^{+} d x \leq \bar{C}
$$

We can then choose, $\sigma=\underline{C} / 8 \bar{C}$, a universal constant. The last conclusion follows from the density Theorem for lower dimensional Hausdorff Measure ([EG], Theorem 1, pp. 72), just by observing that $\mathcal{H}^{N-1}\left\lfloor F\left(u_{0}\right)\right.$ is a Radon Measure.

\section{Special Form of Singular perturbation and Blow-up Convergence Results}

In the previous sections, we have described the "weak" geometry of the free boundary $F\left(u_{0}\right)$ for the limit of the least supersolutions $u_{\varepsilon}$ to the equation $\left(E_{\varepsilon}\right)$. In order to study in more depth the limit free boundary problem, we

will restrict ourselves to deal with the special case where equation $\left(E_{\varepsilon}\right)$ assumes the following form

$$
\Delta u=\beta_{\varepsilon}(u) F(\nabla u) \text { in } \Omega
$$

where $F$ satisfies

$F-1) \quad F \in C^{0,1}\left(\mathbb{R}^{N}\right)$;

$F-2) 0<F_{\text {min }} \leq F(x) \leq F_{\max }<\infty \forall x \in \mathbb{R}^{N}$;

and $\beta$ satisfies the conditions in specified in [CV], i.e,

$\beta-1) \beta \in C^{0,1}(\mathbb{R})$

$\beta-2) \beta>0$ in $(0,1)$ and support of $\beta$ is $[0,1]$;

$\beta-3) \beta$ is increasing in $[0,1 / 2)$ and decreasing in $(1 / 2,1]$;

$\beta-4) \int_{0}^{1} \beta(s) d s:=M>0$

and additionally, $\beta-5) \beta(t) \geq B_{0} t^{+}$for all $t \leq 3 / 4$, where $B_{0}>0$. 
Observe that from the condition $\beta-2$, we conclude that there exists $\tau_{0}>0$ such that

$$
\beta(t) \geq \frac{\tau_{0}}{F_{\min }} \text { for } t \in[1 / 4,3 / 4]
$$

and we define the following universal constant

$$
A_{0}:=\frac{\tau_{0}}{3 N}
$$

As we pointed out in the introduction, the semilinear equations $\left(S E_{\varepsilon}\right)$ have connections with the Prandtl-Batchelor free boundary problems as they were pointed out by Luis Caffarelli, David Jerison and Carlos Kenig in [CJK].

Remark 5.1. From the assumption $F-1$, we can improve the regularity obtained in Theorem $[2.3)$. Indeed, it follows from ([CC], Theorem 8.1) or ([FH], Theorem 5.20) that if $v_{\varepsilon}$ is a continuous viscosity solution to $\left(S E_{\varepsilon}\right)$, then $v_{\varepsilon}$ is actually a classical solution of $\left(S E_{\varepsilon}\right)$.

The presence of the gradient in the equations $\left(S E_{\varepsilon}\right)$ does not affect rescaling properties (see remark (5.5), below). This way, the convergence of blow-up's and their compatibility condition proven in [CLW1] and [LW] are preserved. Since the proofs are a small variant of the original ones, they will be ommited.

Proposition 5.2 (Blow-up convergence - [CLW1], Lemma 3.2). Let $\left\{v_{\varepsilon}\right\}_{\varepsilon>0}$ be a family of viscosity solutions to $\left(S E_{\varepsilon}\right)$. Assume for a subsequence $\varepsilon_{j} \rightarrow 0$, $v_{\varepsilon_{j}} \rightarrow v$ uniformly in compact subsets of $\Omega$. Let $x_{0}, x_{n} \in \Omega \cap \partial\{v>0\}$ be such that $x_{n} \rightarrow x_{0}$ as $k \rightarrow \infty$. Let $\lambda_{n} \rightarrow 0, v_{\lambda_{n}}(x)=\left(1 / \lambda_{n}\right) v\left(x_{n}+\lambda_{n} x\right)$ and $\left(v_{\varepsilon_{j}}\right)_{\lambda_{n}}(x)=\left(1 / \lambda_{n}\right) v_{\varepsilon_{j}}\left(x_{n}+\lambda_{n} x\right)$. Suppose, that $v_{\lambda_{n}} \rightarrow V$ as $n \rightarrow \infty$ uniformly on compact subsets of $\mathbb{R}^{N}$. Then, there exists $j(n) \rightarrow \infty$ such that for every $j_{n} \geq j(n)$ there holds that $\varepsilon_{j_{n}} / \lambda_{n} \rightarrow 0$, and

i) $\left(v_{\varepsilon_{j_{n}}}\right)_{\lambda_{n}} \rightarrow V$ uniformly in compact subsets of $\mathbb{R}^{N}$;

ii) $\nabla\left(v_{\varepsilon_{j_{n}}}\right)_{\lambda_{n}} \rightarrow \nabla V$ in $L_{l o c}^{2}\left(\mathbb{R}^{N}\right)$;

iii) $\nabla v_{\lambda_{n}} \rightarrow \nabla V$ in $L_{l o c}^{2}\left(\mathbb{R}^{N}\right)$.

Proposition 5.3 (Blow-up compatibility condition - [LW], Lemma 3.1). Let $\left\{v_{\varepsilon}\right\}_{\varepsilon>0}$ be a family of viscosity solutions to $\left(S E_{\varepsilon}\right)$. Assume for a subsequence $\varepsilon_{j} \rightarrow 0, v_{\varepsilon_{j}} \rightarrow v$ uniformly in compact subsets of $\Omega$. Let $x_{0} \in F(v)$ and, for $\lambda>0$, let $v_{\lambda}(x)=\frac{1}{\lambda} v\left(x_{0}+\lambda x\right)$. Let $\lambda_{n} \rightarrow 0$ and $\widetilde{\lambda}_{n} \rightarrow 0$ be such that 


$$
\begin{aligned}
& v_{\lambda_{n}} \rightarrow V=\alpha x_{1}^{+}-\gamma x_{1}^{-}+o(|x|), \\
& v_{\widetilde{\lambda_{n}}} \rightarrow \widetilde{V}=\widetilde{\alpha} x_{1}^{+}-\widetilde{\gamma} x_{1}^{-}+o(|x|),
\end{aligned}
$$

uniformly in compact sets of $\mathbb{R}^{N}$, with $\alpha, \widetilde{\alpha}, \gamma, \widetilde{\gamma} \geq 0$. Then $\alpha \gamma=\widetilde{\alpha} \widetilde{\gamma}$.

Definition 5.4. A continuous family $\left\{v_{\varepsilon}\right\}_{\varepsilon>0}$ of viscosity solutions to $\left(S E_{\varepsilon}\right)$ is said to be a family of least viscosity supersolutions to $\left(S E_{\varepsilon}\right)$ in $\Omega$ if for every open set $V \subset \subset \Omega$, we have for every $\varepsilon>0$

$$
v_{\varepsilon} \mid V=\omega_{\varepsilon}^{V}
$$

where

$$
w_{\varepsilon}^{V}(x):=\inf _{w \in \mathcal{S}_{\varepsilon}(V)} w(x)
$$

$\mathcal{S}_{\varepsilon}(V):=\left\{w \in C^{0}(\bar{V}), w\right.$ viscosity supersolution of $\left(S E_{\varepsilon}\right) ; w \geq v_{\varepsilon}$ on $\left.\partial V\right\}$

Clearly, proceeding by Perron's method, as in Theorem 2.3), $\omega_{\varepsilon}^{V}$ is a continuous viscosity solution of $\left(S E_{\varepsilon}\right)$ in $V$. It follows directly from the Theory developed in Theorem 2.3 that $\left\{u_{\varepsilon}\right\}_{\varepsilon>0}$ is a family of least viscosity supersolutions of $\left(S E_{\varepsilon}\right)$.

Remark 5.5. (Transformations that preserves $\left(S E_{\varepsilon}\right)$ )

i) (Rescaling ) Assume that $v$ is a solution to $\left(S E_{\varepsilon}\right)$ in $\Omega$. If $x_{0} \in \Omega$ and $\lambda>0$, let $T_{x_{0}}^{\lambda}(x):=x_{0}+\lambda x$ we define the open set $\Omega_{x_{0}}^{\lambda}=\left(T_{x_{0}}^{\lambda}\right)^{-1}(\Omega)=$ $\left\{x \in \mathbb{R}^{N} \mid x_{0}+\lambda x \in \Omega\right\}$ and the function $\left(v_{x_{0}}\right)_{\lambda}(x):=\frac{1}{\lambda} v\left(T_{x_{0}}^{\lambda}(x)\right)=\frac{1}{\lambda} v\left(x_{0}+\right.$ $\lambda x)$. It is imediate that,$\left(v_{x_{0}}\right)_{\lambda}$ is a solution in $\Omega_{x_{0}}^{\lambda}$ to

$$
\Delta u=\beta_{\frac{\varepsilon}{\lambda}}(u) F(\nabla u)
$$

Conversely, if $w$ is a solution to $\left(S E_{\frac{\varepsilon}{\lambda}}\right)$ in $\Omega_{x_{0}}^{\lambda}$, we define in $\Omega$ the function $\left(w_{x_{0}}\right)^{\lambda}(y):=\lambda w\left(\left(T_{x_{0}}^{\lambda}\right)^{-1}(y)\right)=\lambda w\left(\frac{y-x_{0}}{\lambda}\right)$. Again, it is clear that $\left(w_{x_{0}}\right)^{\lambda}$ is a solution to $\left(S E_{\varepsilon}\right)$.

This way, the correspondences $v \mapsto\left(v_{x_{0}}\right)_{\lambda}$ and $w \mapsto\left(w_{x_{0}}\right)^{\lambda}$ establish a bijection among solutions of $\left(S E_{\varepsilon}\right)$ and $(S E)_{\frac{\varepsilon}{\lambda}}$. Since those maps preserve order, i.e, $v^{1} \leq v^{2} \Longrightarrow\left(v_{x_{0}}^{1}\right)_{\lambda} \leq\left(v_{x_{0}}^{2}\right)_{\lambda}$ and $w^{1} \leq w^{2} \Longrightarrow\left(w_{x_{0}}^{1}\right)^{\lambda} \leq\left(w_{x_{0}}^{2}\right)^{\lambda}$, we conclude: $\left\{v_{\varepsilon}\right\}_{\varepsilon>0}$ is a family of least viscosity supersolution to $\left(S E_{\varepsilon}\right)$ in $\Omega$ if 
and only if $\left\{\left(\left(v_{\varepsilon}\right)_{x_{0}}\right)_{\lambda}\right\}_{\varepsilon>0}$ is a family of least viscosity solutions to $(S E)_{\frac{\varepsilon}{\lambda}}$ in $\Omega_{x_{0}}^{\lambda}$.

ii) (Invariance under translations) Since the equation $\left(S E_{\varepsilon}\right)$ does not dependend on $x$, the equation is translation invariant, i.e, translations of solutions (subsolutions, supersolutions) $u, v=u(\cdot+h), h \in \mathbb{R}^{N}$ are still solutions (subsolutions, supersolutions) respectively.

\section{Some Qualitative Results}

In this section, we will prove some results that will be used in a decisive way to obtain the classifications of global profiles later on. We will start with some definitons.

Definition 6.1. We set for $\sigma>0$ the scaled function

$$
\mathcal{E}_{\sigma}(\beta)(x):=\sigma \beta\left(\frac{x}{\sigma}-\frac{1}{2 \sigma}+\frac{1}{2}\right)
$$

Geometrically, the graph of $\mathcal{E}_{\sigma}(\beta)$ corresponds to a $\sigma$ - rescaling of the graph of $\beta$ with respect to $x=\frac{1}{2}$. So, $\operatorname{supp}\left(\mathcal{E}_{\sigma}(\beta)\right)=\left[\kappa_{\sigma}^{-}, \kappa_{\sigma}^{+}\right]$, where, $\kappa_{\sigma}^{-}:=\frac{1}{2}-\frac{\sigma}{2}$ and $\kappa_{\sigma}^{+}:=\frac{1}{2}+\frac{\sigma}{2}$. Also, for any $\sigma>0, \mathcal{E}_{\sigma}(\beta) \in C^{0,1}(\mathbb{R})$ with $\operatorname{Lip}\left(\mathcal{E}_{\sigma}(\beta)\right)=\operatorname{Lip}(\beta)$. By $\beta-3)$, it is easy to verify that

$$
\begin{aligned}
& 0<\sigma<1 \Longrightarrow \mathcal{E}_{\sigma}(\beta)(t)<\beta(t) \text { for } t \in \operatorname{supp}\left(\mathcal{E}_{\sigma}(\beta)\right) \\
& \sigma>1 \Longrightarrow \mathcal{E}_{\sigma}(\beta)(t)>\beta(t) \text { for } t \in[0,1]=\operatorname{supp}(\beta)
\end{aligned}
$$

Moreover, from the relation

$$
\sigma_{1}, \sigma_{2}>0 \Longrightarrow \mathcal{E}_{\frac{\sigma_{2}}{\sigma_{1}}}\left(\mathcal{E}_{\sigma_{1}}(\beta)\right)=\mathcal{E}_{\sigma_{2}}(\beta)
$$

It follows that

$$
0<\sigma_{1}<\sigma_{2} \Longrightarrow \mathcal{E}_{\sigma_{1}}(\beta)(t)<\mathcal{E}_{\sigma_{2}}(\beta)(t) \text { for } t \in \operatorname{supp}\left(\mathcal{E}_{\sigma_{1}}(\beta)\right)
$$

We set,

$$
M_{\sigma}:=\int_{\kappa_{\sigma}^{-}}^{\kappa_{\sigma}^{+}} \mathcal{E}_{\sigma}(\beta)(t) d t=\sigma^{2} M
$$


As usual, we use the same notation for the $\varepsilon$-rescaling, i.e,

$$
\left(\mathcal{E}_{\sigma}(\beta)\right)_{\varepsilon}(t)=\frac{1}{\varepsilon} \mathcal{E}_{\sigma}(\beta)\left(\frac{t}{\varepsilon}\right) .
$$

Let us also define, for $|\mu|<F_{\min } / 2$ and $|\delta|<\frac{1}{2}$,

$$
F_{\delta, \mu}(p)=(1+\delta)(F(p)+\mu)>\frac{\delta F_{\min }}{4}>0
$$

and finally, let $e_{1}=(1,0, \ldots, 0)$ be the first canonical vector in $\mathbb{R}^{N}$,

$$
H_{\delta, \mu}(t)=\int_{0}^{t} \frac{s}{\left(F_{\delta, \mu}\left(s e_{1}\right)\right)} d s=\int_{0}^{t} \frac{s}{(1+\delta)\left(F\left(s e_{1}\right)+\mu\right)} d s
$$

We also denote,

$$
H(t)=H_{0,0}(t)=\int_{0}^{t} \frac{s}{F\left(s e_{1}\right)} d s
$$

In the next Lemma, we show that the monotonicity relation in (6.2) still holds if we perturb $\mathcal{E}_{\sigma}(\beta)$ by a scaling factor close enough to 1 .

Lemma 6.2. Assume $0<\sigma_{1}<\sigma_{2}$. If $\theta$ is close enough to 1 , then for every $\varepsilon>0$ we have the following inequalities

$$
\begin{aligned}
& \left(\mathcal{E}_{\sigma_{2}}(\beta)\right)_{\varepsilon}(t) \geq\left(\mathcal{E}_{\sigma_{1}}(\beta)\right)_{\varepsilon}(\theta t) \quad \text { for all } t \in \mathbb{R} \\
& \left(\mathcal{E}_{\sigma_{2}}(\beta)\right)_{\varepsilon}(\theta t) \geq\left(\mathcal{E}_{\sigma_{1}}(\beta)\right)_{\varepsilon}(t) \quad \text { for all } t \in \mathbb{R}
\end{aligned}
$$

Proof. Clearly, by rescaling, it is enough to prove the Lemma for $\varepsilon=1$. So, let us define the following functions in $\mathbb{R}$,

$$
\begin{aligned}
G_{\theta}(t) & =\mathcal{E}_{\sigma_{2}}(\beta)(\theta t)-\mathcal{E}_{\sigma_{1}}(\beta)(t) \\
J_{\theta}(t) & =\mathcal{E}_{\sigma_{2}}(\beta)(t)-\mathcal{E}_{\sigma_{1}}(\beta)(\theta t)
\end{aligned}
$$

We will prove that $G_{\theta}, J_{\theta} \geq 0 \forall t \in \mathbb{R}$. Indeed, let $K \subset \mathbb{R}$ be a compact interval such that supp $\mathcal{E}_{\sigma_{1}}(\beta) \subsetneq K \subsetneq \operatorname{supp} \mathcal{E}_{\sigma_{2}}(\beta)$. Setting $G(t)=\mathcal{E}_{\sigma_{2}}(\beta)(t)-$ $\mathcal{E}_{\sigma_{1}}(\beta)$, since $\mathcal{E}_{\sigma}(\beta)(t)$ is Lipschitz continuous for $\sigma>0$, we have $G_{\theta} \rightarrow G$ and $J_{\theta} \rightarrow G$ locally uniformly in compact subsets of $\mathbb{R}$. By, 6.2), $G>0$ in $K$. In particular, by the uniform convergence, $G_{\theta}, J_{\theta}>0$ in $K$ for $\theta$ close enough to 1. By the other hand, clearly, $G_{\theta}(t) \geq 0$ for $t \notin K$. If $g_{\theta}(t)=\mathcal{E}_{\sigma_{1}}(\beta)(\theta t)$ then 
for $\theta$ close enough to 1 , supp $g_{\theta}=\frac{1}{\theta}\left(\operatorname{supp} \mathcal{E}_{\sigma_{1}}(\beta)\right) \subsetneq K$ and thus, $J_{\theta}(t) \geq 0$ for $t \notin K$. This finishes the Lemma.

Now, we prove a Lemma that says essentially that if a "almost" strict subsoluton to $\left(S E_{\varepsilon}\right)$ is above a supersolution to $\left(S E_{\varepsilon}\right)$, then they cannot touch inside the domain. This Lemma will be used later on with the help of some barriers to prevent the slopes of the blow-up limits to have a "too closed" aperture.

Lemma 6.3 (No interior contact). Let $u_{1}, u_{2} \in C^{2}\left(B_{1}\right) \cap C^{0}\left(\bar{B}_{1}\right)$ and $\sigma>1$ such that

$$
\begin{gathered}
\Delta u_{1} \leq \beta_{\varepsilon}\left(u_{1}\right) F\left(\nabla u_{1}\right) \text { in } B_{1} \\
\Delta u_{2} \geq\left(\mathcal{E}_{\sigma}(\beta)\right)_{\varepsilon}\left(u_{2}\right) F\left(\nabla u_{2}\right) \text { in } B_{1} \\
u_{1} \geq u_{2} \text { in } B_{1}
\end{gathered}
$$

Then, $u_{2}$ cannot touch $u_{1}$ in an interior point.

Proof. Let us prove the renormalized case $\varepsilon=1$. The general case will follow analogously. So, let us assume, by contradiction, that $u_{2}$ touches $u_{1}$ by below at $x_{0} \in B_{1}$. This way $\Delta u_{2}\left(x_{0}\right) \leq \Delta u_{1}\left(x_{0}\right)$. Moreover, since $\nabla u_{1}\left(x_{0}\right)=\nabla u_{2}\left(x_{0}\right)$ and $\mathcal{E}_{\sigma}(\beta) \geq \beta(\sigma>1)$, we have the opposite inequality and thus

$$
\Delta u_{2}\left(x_{0}\right)=\Delta u_{1}\left(x_{0}\right)
$$

If we choose $1<\bar{\sigma}<\sigma$, then $\beta \leq \mathcal{E}_{\bar{\sigma}}<\mathcal{E}_{\sigma}$ in supp $\mathcal{E}_{\bar{\sigma}}=[a, b]$, where $a<0$ and $b>1$. Thus, $c=u_{1}\left(x_{0}\right)=u_{2}\left(x_{0}\right) \notin[a, b]$. Let us suppose $c>b$. Consider $r=\operatorname{dist}\left(x_{0},\left\{u_{1} \leq \frac{1+b}{2}\right\}\right)$ and consider the convex set $A=\bar{B}_{r}\left(x_{0}\right) \cap \bar{B}_{1}$. Since $u_{1}$ is harmonic in $A^{\circ}, u_{2}$ is subharmonic in $A^{\circ}$ and $A^{\circ}$ is connected, the strong maximum principle implies $u_{1} \equiv u_{2}$ in $A$. In particular, $\nabla u_{1} \equiv \nabla u_{2}$ and $\Delta u_{1} \equiv \Delta u_{2}$ in $A^{\circ}$. If $x_{1}$ is such that $r=\left|x_{1}-x_{0}\right|$, then $u_{1}\left(x_{1}\right)=\frac{1+b}{2}$. This way, the segment $\left(x_{1}, x_{0}\right) \subset A^{\circ}$. In particular, by the mean value Theorem, we can find $x_{2}$ in the open segment, for which $\frac{1+b}{2}<u_{1}\left(x_{2}\right)=\frac{1+b}{2}+\frac{b-1}{8}=\bar{b}<b$. This way, since $x_{2} \in A^{\circ}$, we have $u_{1}\left(x_{2}\right)=\bar{b}=u_{2}\left(x_{2}\right), \nabla u_{1}\left(x_{2}\right)=p=\nabla u_{2}\left(x_{2}\right)$ and $\Delta u_{1}\left(x_{2}\right)=\Delta u_{2}\left(x_{2}\right)$. Thus,

$$
\beta(\bar{b}) F(p)=\Delta u_{1}\left(x_{2}\right)=\Delta u_{2}\left(x_{2}\right)=\mathcal{E}_{\sigma}(\beta)(\bar{b}) F(p)
$$


which implies, since $F>0, \beta(\bar{b})=\mathcal{E}_{\sigma}(\bar{b})$, a contraditcion since $\bar{b} \in(a, b)$. If $c \leq a$ we proceed similarly. So, $u_{2}$ never touches $u_{1}$ and the Lemma is proven.

In the next Proposition, we contruct a radially symmetric supersolution to $\left(S E_{\varepsilon}\right)$ where its value in a inner disk is much smaller that its value on the boundary. This will be used to prove that the least supersolution $u_{\varepsilon}$ have expontential decay inside the domain.

Proposition 6.4 (Radially symmetric supersolution). Given $\eta>0$, there exists radially symmetric functions $\Theta_{\varepsilon} \in C^{1}\left(\mathbb{R}^{N}\right) \cap W_{\text {loc }}^{2, \infty}\left(\mathbb{R}^{N}\right)$ and universal constants $\kappa_{2}>0$ and $0<\kappa_{1}<1$ such that

i) $\Theta_{\varepsilon} \equiv \frac{\varepsilon}{4}$ in $B_{\kappa_{1} \eta}$

ii) $\Theta_{\varepsilon} \geq \kappa_{2} \eta$ in $\mathbb{R}^{N} \backslash B_{\eta}$

iii) $\Theta_{\varepsilon}$ is a a viscosity supersolution to $\left(S E_{\varepsilon}\right)$ for $\varepsilon$ small enough.

Proof. We will work assuming first that $\varepsilon=1$. After that, we will rescale the construction to obtain $\Theta_{\varepsilon}$. Let $L \geq \frac{10}{\sqrt{2 A_{0}}}$, we define,

$$
\bar{\Theta}(r)=\left\{\begin{aligned}
1 / 4, & \text { for } 0 \leq r \leq L \\
G(r)=A_{0}(r-L)^{2}+1 / 4 & \text { for } L \leq r \leq L+1 / \sqrt{2 A_{0}} \\
\Gamma(r) & \text { for } r \geq L+1 / \sqrt{2 A_{0}}
\end{aligned}\right.
$$

where $\Gamma$ solves

$$
\begin{gathered}
\Gamma_{r r}+\frac{N-1}{r} \Gamma_{r}=0 \text { for } r \geq L+1 / \sqrt{2 A_{0}} \\
\Gamma\left(L+1 / \sqrt{2 A_{0}}\right)=3 / 4, \quad \Gamma_{r}\left(L+1 / \sqrt{2 A_{0}}\right)=\sqrt{2 A_{0}}
\end{gathered}
$$

Let us assume $N \geq 3$. Then

$$
\begin{gathered}
\Gamma(r)=3 / 4+\frac{\sqrt{2 A_{0}}}{N-2}\left(L+1 / \sqrt{2 A_{0}}\right)-\frac{\sqrt{2 A_{0}}}{N-2}\left(L+1 / \sqrt{2 A_{0}}\right)^{N-1} r^{2-N}= \\
=K_{L}-f(r), \text { respectively. }
\end{gathered}
$$

This way,

$$
\kappa^{2-N}<\frac{1}{2}\left(\frac{10}{11}\right)^{N-1} \Rightarrow \kappa^{2-N}<\left(\frac{10}{11}\right)^{N-1} \frac{1}{2 L}\left(L+1 / \sqrt{2 A_{0}}\right) \Longrightarrow
$$




$$
\Longrightarrow \frac{\sqrt{2 A_{0}}}{N-2}\left(\frac{11}{10}\right)^{N-1} \kappa^{2-N} L \leq \frac{1}{2} \frac{\sqrt{2 A_{0}}}{N-2}\left(L+1 / \sqrt{2 A_{0}}\right)
$$

Translating the inequality above in terms of $K_{L}$ and $f(r)$, and recalling that $L>\frac{10}{\sqrt{2 A_{0}}}$

$$
f\left(\kappa_{3} L\right) \leq \frac{1}{2} K_{L} \quad \text { for } \kappa_{3}^{2-N}=\frac{1}{4}\left(\frac{10}{11}\right)^{N-1}<1
$$

In particular, since $\Gamma$ is increasing, $\Gamma(r)>\frac{1}{2} K_{L} \geq \kappa_{4} L$ for $r \geq \kappa_{3} L$, where $\kappa_{4}=\sqrt{2 A_{0}} / 2(N-2) L$. Finally, let us observe, that for $r \in\left(L, L+1 / \sqrt{2 A_{0}}\right)$, $1 / 4 \leq \Theta \leq 3 / 4$, so

$$
\Theta_{r r}+\frac{N-1}{r} \Theta_{r}=G_{r r}+\frac{N-1}{r} G_{r} \leq 2 A_{0} N \leq \tau_{0} \leq \beta(\Theta(r)) F\left(\Theta_{r}(r) \frac{x}{|x|}\right)
$$

Thus, setting $\Theta(x):=\bar{\Theta}(|x|)$, by construction, $\Theta \in C^{1}\left(\mathbb{R}^{N}\right) \cap W_{\text {loc }}^{2, \infty}\left(\mathbb{R}^{N}\right)$ is a $L_{\text {loc }}^{\infty}$-strong solution to the equation (i.e, it belongs to $W_{\text {loc }}^{2, \infty}\left(\mathbb{R}^{N}\right)$ and solves the equation a.e.)

$$
\begin{gathered}
\Delta u=\beta(u) F(\nabla u) \\
\text { if } \varepsilon<\varepsilon_{0}:=\frac{\eta \sqrt{2 A_{0}}}{10 \kappa_{3}} \text {, we can find } L>\frac{10}{\sqrt{2 A_{0}}} \text { such that } \varepsilon=\frac{\eta}{\kappa_{3} L} \text { and defining } \\
\Theta_{\varepsilon}(x):=\varepsilon \Theta\left(\frac{x}{\varepsilon}\right)
\end{gathered}
$$

We see that $\Theta_{\varepsilon} \in C^{1}\left(\mathbb{R}^{N}\right) \cap W_{l o c}^{2, \infty}\left(\mathbb{R}^{N}\right)$ and $\left.i\right)$ and $\left.i i\right)$ are satisfied with $k_{1}=$ $1 / \kappa_{3}$ and $\kappa_{2}=\kappa_{4} / \kappa_{3}$. The fact the $\Theta_{\varepsilon}$ are viscosity solutions of $\left(S E_{\varepsilon}\right)$ follows from Theorem (2.1) in CKSS] or more generally by the results in [CCKS]. The case $N=2$, where $\Gamma(r)=3 / 4+\sqrt{2 A_{0}}\left(L+1 / \sqrt{2 A_{0}}\right) \log \left(\frac{r}{L+\sqrt{2 A_{0}}}\right)$, is proven similarly.

We will prove a interesting geometric property of family of least supersolution to $\left(S E_{\varepsilon}\right)$. Essentially, it says that if they are small in a certain domain, as soon as we get a little bit inside the domain, they become much smaller, decaying exponentially fast with $\varepsilon$.

In the next proposition, we use the notation $Q_{r}=\left\{\left(x_{1}, x^{\prime}\right) \in \mathbb{R}^{N} ;\left|x_{1}\right| \leq r,\left|x^{\prime}\right| \leq r\right\}$ 
Proposition 6.5 (Expontential decay inside). Suppose $\left\{v_{\varepsilon}\right\}_{\varepsilon>0}$ is a family of least supersolutions of $\left(S E_{\varepsilon}\right)$ and that for some $\eta>0$ (small), $\left\|v_{\varepsilon}^{+}\right\|_{L^{\infty}\left(Q_{1}\right)}<$ $\kappa_{2} \eta$. Then, there exist a constant $C_{\eta}>0$ depending on $\eta$ such that

$$
v_{\varepsilon}^{+}(x) \leq C_{\eta} \varepsilon^{3} \text { for all } x \in Q_{1-2 \eta} \text { and } \varepsilon \text { small enough. }
$$

Proof. Indeed, if $x_{0} \in Q_{1-\eta}, B_{\eta}\left(x_{0}\right) \subset Q_{1}$. We can now place the radially symmetric barrier constructed in the previous Proposition (6.4) in this ball, and since $v_{\varepsilon}$ is the least supersolution of $\left(S E_{\varepsilon}\right)$, we conclude, $v_{\varepsilon}\left(x_{0}\right) \leq \frac{\varepsilon}{4}$. This way,

$$
v_{\varepsilon}(x) \leq \frac{\varepsilon}{4} \text { for all } x \in Q_{1-\eta}
$$

Let us denote by $G_{x}$ the positive Green's function of the ball $B_{\eta}(x)$. If $x_{1} \in Q_{1-2 \eta}, \bar{B}_{\eta}\left(x_{1}\right) \subset Q_{1-\eta}$. Using the Green's representation formula

$$
v_{\varepsilon}\left(x_{1}\right)=f_{\partial B_{\eta}\left(x_{1}\right)} v_{\varepsilon} d \mathcal{H}^{N-1}-\int_{B_{\eta}\left(x_{1}\right)} G_{x_{1}}(y) \Delta v_{\varepsilon}(y) d y
$$

We have by property $\beta-5)$,

$$
\frac{F_{\min } B_{0}}{\varepsilon^{2}}\left(\inf _{B_{\frac{\eta}{2}}\left(x_{1}\right)} G_{x_{1}}\right) \int_{B_{\eta / 2}\left(x_{1}\right)} v_{\varepsilon}^{+}(y) d y \leq F_{\min } \int_{B_{\eta / 2}\left(x_{1}\right)} G_{x_{1}}(y) \beta_{\varepsilon}\left(v_{\varepsilon}(y)\right) \leq \frac{\varepsilon}{2}
$$

Since $v_{\varepsilon}^{+}$is subharmonic,

$$
v_{\varepsilon}^{+}\left(x_{1}\right) \leq \int_{B_{\eta / 2}\left(x_{1}\right)} v_{\varepsilon}^{+}(y) d y
$$

Recalling that $\inf _{B_{\eta / 2}\left(x_{1}\right)} G_{x_{1}}=A_{\eta}$, where $A_{\eta}$ is a universal constant depending on $\eta$ and combining 6.11 and 6.12, we have

$$
v_{\varepsilon}^{+}\left(x_{1}\right) \leq \frac{\varepsilon^{3}}{2 F_{\min } B_{0} A_{\eta}\left|B_{\eta / 2}\left(x_{1}\right)\right|}=C_{\eta} \varepsilon^{3}
$$

Finally, to end this section, we study the 1-dimensional profiles of our family of regularizing equations. This profiles will be modified in the next section, to create barries with uniformly curved free boundaries. 
Lemma 6.6 (1-dimensional profiles). Assume that $P \in C^{2}(\mathbb{R})$ is the unique solution of

$$
\begin{gathered}
u_{s s}=\mathcal{E}_{\sigma}(\beta)(u) F_{\delta, \mu}\left(u_{s} e_{1}\right)=(1+\delta)\left(\mathcal{E}_{\sigma}(\beta)(u)\right)\left(F\left(u_{s} e_{1}\right)+\mu\right) \\
u(0)=\kappa_{\sigma}^{+} \text {and } u_{s}(0)=\alpha>0
\end{gathered}
$$

a) If $\gamma \geq 0$ and $H_{\delta, \mu}(\alpha)-H_{\delta, \mu}(\gamma)>M_{\sigma}$, there exist $\bar{\gamma}>\gamma$ and $\bar{s}<0$ depending on $\alpha, \gamma, \delta, \sigma, \mu$ such that

$$
P(s)=\left\{\begin{array}{r}
\kappa_{\sigma}^{+}+\alpha s, \quad s \geq 0 \\
\bar{\gamma}(s-\bar{s})+\kappa_{\sigma}^{-}, \quad s \leq \bar{s},
\end{array}\right.
$$

b) If $\gamma \geq 0$ with $H_{\delta, \mu}(\alpha)-H_{\delta, \mu}(\gamma)<M_{\sigma}$ we have two cases:

b.1) If $H_{\delta, \mu}(\alpha)>M_{\sigma}$, there exist $\bar{\gamma}<\gamma$ and $\bar{s}<0$ depending on $\alpha, \gamma, \delta, \sigma, \mu$ such that

$$
P(s)=\left\{\begin{array}{r}
\kappa_{\sigma}^{+}+\alpha s, \quad s \geq 0 \\
\bar{\gamma}(s-\bar{s})+\kappa_{\sigma}, \quad s \leq \bar{s},
\end{array}\right.
$$

or

b.2) If $H_{\delta, \mu}(\alpha)<M_{\sigma}$, there exist $\bar{\gamma}>0$ and $\bar{s}<0$ depending on $\alpha, \gamma, \delta, \sigma, \mu$ such that

$$
P(s)=\left\{\begin{array}{rc}
\kappa_{\sigma}^{+}+\alpha s, \quad s \geq 0 \\
\kappa_{\sigma}^{+}-\bar{\gamma}(s-\bar{s}) \quad s \leq \bar{s}
\end{array}\right.
$$

Moreover, in this case, there exists $\bar{\kappa}_{\sigma}$ such that $\kappa_{\sigma}^{-}<\bar{\kappa}_{\sigma}<P(s)<\kappa_{\sigma}^{+}$for $\bar{s}<s<0$. Furthermore, setting $P_{\varepsilon}(s)=\varepsilon P\left(\frac{s}{\varepsilon}\right)$, it solves

$$
\begin{array}{r}
u_{s s}=\left(\mathcal{E}_{\sigma}(\beta)\right)_{\varepsilon}(u) F_{\delta, \mu}\left(u_{s} e_{1}\right) \\
u(0)=\varepsilon \kappa_{\sigma}^{+} \text {and } u_{s}(0)=\alpha>0
\end{array}
$$

Proof. We start by observing that $H_{\delta, \mu}$ is a bijection from $[0,+\infty)$ over itself. This follows since $H_{\delta, \mu}(s) \geq \frac{s^{2}}{3 F_{\max }}$, and $\left(H_{\delta, \mu}\right)_{s}>0$ for $s>0$. Multiplying the equation 6.13 by $P_{s}$ we find,

$$
\left(H_{\delta, \mu}\left(P_{s}\right)\right)_{s}=B^{\sigma}(P)_{s}
$$


where $B^{\sigma}(\zeta)=\int_{\kappa_{\sigma}^{-}}^{\zeta} \mathcal{E}_{\sigma}(\beta)(t) d t$. Integrating this equation, we obtain, in cases a) and $b .1$ ), for some $\bar{\gamma}>0$,

$$
H_{\delta, \mu}\left(P_{s}(s)\right)-B^{\sigma}(P(s))=H_{\delta, \mu}(\alpha)-M_{\sigma}=H_{\delta, \mu}(\bar{\gamma})>0
$$

This way, since from the expression above, $P_{s} \geq 0$

$$
0<\bar{\gamma} \leq P_{s}(s) \leq \alpha, \quad \text { for } t \in \mathbb{R}
$$

In case $a$ ), we have $H_{\delta, \mu}(\bar{\gamma})>H_{\delta, \mu}(\gamma) \geq 0$ and so $\bar{\gamma}>\gamma$. In case $b$ ), $H_{\delta, \mu}(\bar{\gamma})<H_{\delta, \mu}(\gamma)$, and thus $\bar{\gamma}<\gamma$. From the inequality 6.17) above, the conclusion of $a$ ) and $b .1$ ) is straightforward. To prove $b .2$ ), we observe again, the relation 6.17 , i.e,

$$
H_{\delta, \mu}\left(P_{s}(s)\right)-B^{\sigma}(P(s))=H_{\delta, \mu}(\alpha)-M_{\sigma}<0
$$

Since $\bar{P}_{s s} \geq 0, \bar{P}_{s}$ is nondecreasing, thus, $P_{s}>0$ in $s \geq 0$. This way, we conclude, $P(s)=\kappa_{\sigma}^{+}+\alpha s$ for $s \geq 0$. Observing that, $H_{\delta, \mu} \geq 0$, we see that relation 6.18, implies, in particular, $P>0$ in $\mathbb{R}$. Actually, $\inf _{\mathbb{R}} P=\bar{\kappa}_{\sigma}>\kappa_{\sigma}^{-}$, otherwise, we could take a minimizing sequence $s_{n}$, 6.18 would provide

$$
0 \leq H_{\delta, \mu}\left(P_{s}\left(s_{n}\right)\right)=B^{\sigma}\left(P\left(s_{n}\right)\right)+H(\alpha)-M
$$

letting $n \rightarrow \infty$, we would obtain a contradiction. Our assetion will follow, if we can show that $\lim _{s \rightarrow-\infty} P(s)=+\infty$. For this purpose, it is enough to show that there exists $b<0$ such that $P_{s}(b)<0$, since by convexity we have

$$
P(s) \geq P(b)+P_{s}(b)(s-b) \forall s \in \mathbb{R}
$$

So, let us suppose by contradiction, that $P_{s} \geq 0$ for all $s \in \mathbb{R}$. This way, $P$ is nondecreasing and thus $\lim _{s \rightarrow-\infty} P(s)=\bar{\kappa}_{\sigma}>\kappa_{\sigma}^{-}$and $\lim _{s \rightarrow-\infty} P_{s}(s)=0$. Applying limit as $s \rightarrow-\infty$ in 6.18), we find

$$
0<B^{\sigma}\left(\bar{\kappa}_{\sigma}\right)=M_{\sigma}-H_{\delta, \mu}(\alpha)=\rho<M_{\sigma}
$$

Since $B^{\sigma}$ is invertible in $\left(\kappa_{\sigma}^{-}, \kappa_{\sigma}^{+}\right)$, we conclude that $P(s) \rightarrow \bar{\kappa}_{\sigma} \in\left(\kappa_{\sigma}^{-}, \kappa_{\sigma}^{+}\right)$as $s \rightarrow-\infty$. In particular, for $\eta>0$ small enough, $P(s) \in A_{\eta}=\left[\bar{\kappa}_{\sigma}-\eta, \bar{\kappa}_{\sigma}+\eta\right] \subsetneq$ $\left(\kappa_{\sigma}^{-}, \kappa_{\sigma}^{+}\right)$for $s \leq c, c<0$. This way, if $\tau=i n f_{A_{\eta}} \mathcal{E}_{\sigma}(\beta)$, we have 


$$
P_{s s}(s)=\left(\mathcal{E}_{\sigma}(\beta)\right)(P(s)) F_{\delta, \mu}\left(P_{s}(s) e_{1}\right) \geq \tau \frac{F_{\text {min }}}{4}>0 \text { for all } s \leq c
$$

But, this implies $P_{s}(c)=\infty$, clearly a contradiction since, $P \in C^{2}(\mathbb{R})$. This finishes $b .2$ ).

\section{Slope Barriers with Curved Free Boundary}

In this section, we will construct some barriers with uniformly curved free boundaries. The are essentially obtained by a uniform bending of the 1dimensional profiles given by Lemma (6.6). The key tool used to acomplish this is a sequence of Kelvin transforms with respect to large spheres, i.e, spheres having centers and radii approaching infinity. These barriers will be the fundamental ingridient to classify global profiles (2-plane functions) in the next section.

Remark 7.1. For later reference, we will recall some facts about Inversion and Kelvin transform that will be used in the sequel. For $L>0$, we denote

$$
\begin{aligned}
& \mathbb{S}_{L}=\left\{x \in \mathbb{R}^{N} ;\left|x+L e_{1}\right|=L\right\} \\
& \mathbb{S}_{L}^{\star}=\left\{x \in \mathbb{R}^{N} ;\left|x-L e_{1}\right|=L\right\}
\end{aligned}
$$

The Kelvin transforms of a continuous function $u$ with respect to $\mathbb{S}_{L}$ and $\mathbb{S}_{L}^{\star}$ are given, respectively, by $K_{L}$ and $T_{L}$ below

$$
K_{L}[u](x)=\left(\rho_{L}(x)\right)^{N-2} u\left(I_{L}(x)\right)
$$

where $I_{L}, J_{L}$ are the inversions with respect to $\mathbb{S}_{L}$ and $\mathbb{S}_{L}^{\star}$, respectively, given by

$$
\begin{gathered}
I_{L}(x)=-L e_{1}+\frac{L^{2}}{\left|x+L e_{1}\right|^{2}}\left(x+L e_{1}\right) \\
J_{L}(x)=L e_{1}+\frac{L^{2}}{\left|x-L e_{1}\right|^{2}}\left(x-L e_{1}\right)
\end{gathered}
$$




$$
\rho_{L}(x)=\frac{L}{\left|x+L e_{1}\right|} \quad \text { and } \quad \varrho_{L}(x)=\frac{L}{\left|x-L e_{1}\right|}
$$

it follows also that,

$$
\begin{aligned}
& \Delta K_{L}[u](x)=\left(\rho_{L}(x)\right)^{N+2} \Delta u\left(I_{L}(x)\right) \\
& \Delta T_{L}[u](x)=\left(\varrho_{L}(x)\right)^{N+2} \Delta u\left(J_{L}(x)\right)
\end{aligned}
$$

Furthermore, if $\mathcal{R}_{1}$ is the orthogonal reflection with respect the hyperplane $\left\{x_{1}=0\right\}$, then for any $L_{0}>0$, and $L>L_{0}$ we have

$$
\begin{gathered}
\rho_{L} \rightarrow 1 \text { in } C_{l o c}^{1}\left(\mathbb{R}^{N} \backslash\left\{l e_{1} ; l \leq-10 L_{0}\right\}\right) \\
\varrho_{L} \rightarrow 1 \text { in } C_{l o c}^{1}\left(\mathbb{R}^{N} \backslash\left\{l e_{1} ; l \geq 10 L_{0}\right\}\right) \\
I_{L} \rightarrow \mathcal{R}_{1} \text { in } C_{l o c}^{1}\left(\mathbb{R}^{N} \backslash\left\{l e_{1} ; l \leq-10 L_{0}\right\}\right) \\
J_{L} \rightarrow \mathcal{R}_{1} \text { in } C_{l o c}^{1}\left(\mathbb{R}^{N} \backslash\left\{l e_{1} ; l \geq 10 L_{0}\right\}\right)
\end{gathered}
$$

For more details about Inversions and Kelvin transforms, check $([\mathrm{B}])$ and $([\mathrm{ABR}])$.

In what follows, we use the cylinder for $L_{0}>0$,

$$
Q_{L_{0}}=\left\{x=\left.\left(x_{1}, x^{\prime}\right) \in \mathbb{R}^{N}|| x\right|_{\infty}=\max \left\{\left|x_{1}\right|,\left|x^{\prime}\right|\right\} \leq 4 L_{0}\right\}
$$

Proposition 7.2 (Above condition barrier). Suppose, $\bar{\sigma}>\sigma>1, \delta, \mu>0$ and $\bar{\alpha}>0, \gamma \geq 0$ are such that $H_{\delta, \mu}(\bar{\alpha})-H_{\delta, \mu}(\gamma)>M_{\bar{\sigma}}$. There exists $\vartheta_{\varepsilon} \in C^{2}\left(Q_{L_{0}}\right)$ such that

a) $\Delta \vartheta_{\varepsilon}(x) \geq\left(\mathcal{E}_{\sigma}(\beta)\right)_{\varepsilon}\left(\vartheta_{\varepsilon}(x)\right) F\left(\nabla \vartheta_{\varepsilon}(x)\right)$ for $x \in Q_{L_{0}}$; 
b) one has

$$
\begin{gathered}
\vartheta_{\varepsilon}<0 \text { in } Q_{L_{0}} \cap \mathbb{B}^{C} \\
\vartheta_{\varepsilon}>0 \text { in } Q_{L_{0}} \cap \mathbb{B}^{\circ} \\
\vartheta_{\varepsilon}=0 \text { on } Q_{L_{0}} \cap \mathbb{S} \\
\mathbb{S} \cap \partial Q_{L_{0}} \subset\left\{x_{1}=d\right\}, \quad d=d\left(\text { radius of } \mathbb{S}, L_{0}\right)>0
\end{gathered}
$$

where $\mathbb{S}=\partial \mathbb{B}$, and $\mathbb{B}$ is a closed ball completely contained in the half space $\left\{x_{1} \geq 0\right\}$, centered in the positive semi-axis generated by $e_{1}$ and tangent to the hyperplane $\left\{x_{1}=0\right\}$;

c) There exists $\widetilde{\alpha}>\bar{\alpha}>\widetilde{\gamma}>\gamma$ such that for $\mathcal{W}(x)=\widetilde{\alpha} x_{1}^{+}-\widetilde{\gamma} x_{1}^{-}$, we have

$$
\begin{gathered}
\mathcal{W}(x) \geq \vartheta_{\varepsilon}(x) \text { in } Q_{L_{0}} \text { and } \mathcal{W}(0)=\vartheta_{\varepsilon}(0) \\
\mathcal{W}\left(x-d e_{1}\right) \geq \vartheta_{\varepsilon}(x) \text { for } x \in Q_{L_{0}} \cap\left\{x=\left(x_{1}, x^{\prime}\right) \in \mathbb{R}^{N} ;\left|x^{\prime}\right|=L_{0}\right\} \\
Q_{\varepsilon} \leq \vartheta_{\varepsilon} \text { along span }\left\{e_{1}\right\}
\end{gathered}
$$

where $Q_{\varepsilon}(x):=\bar{Q}_{\varepsilon}\left(x_{1}\right)$ and $\bar{Q}_{\varepsilon}(s):=P_{\varepsilon}\left(s+a_{\varepsilon}\right)$ is the solution to $\mathcal{E}_{\bar{\alpha}, \delta, \mu, \sigma}$ and $a_{\varepsilon}$ is chosen such that $\bar{Q}_{\varepsilon}(0)=0$. Moreover, $\widetilde{\alpha}$ can be taken as close as we wish from $\alpha$.

Proof. As suggested in $c$ ), let us define

$$
Q_{\varepsilon}(x):=\bar{Q}_{\varepsilon}\left(x_{1}\right)
$$

and recall that $\mathcal{R}_{1}$ denotes the reflection with respect the hyperplane $\left\{x_{1}=0\right\}$. Taking $L>20 L_{0}$, we set

$$
\vartheta_{\varepsilon}^{L}(x):=\left(K_{L}\left[Q_{\varepsilon}\right] \circ \mathcal{R}_{1}\right)(x)=K_{L}\left[Q_{\varepsilon}\right]\left(\mathcal{R}_{1}(x)\right)=\left(\bar{\rho}_{L}(x)\right)^{N-2} Q_{\varepsilon}\left(\bar{I}_{L}(x)\right)
$$

where

$$
\bar{I}_{L}=I_{L} \circ \mathcal{R}_{1}, \bar{\rho}_{L}=\rho \circ \mathcal{R}_{1}
$$

By remark (7.1),

$$
\Delta \vartheta_{\varepsilon}^{L}(x)=\left(\Delta K_{L}\left[Q_{\varepsilon}\right] \circ \mathcal{R}_{1}\right)(x)=\Delta K_{L}\left[Q_{\varepsilon}\right]\left(\mathcal{R}_{1}(x)\right)=
$$




$$
=\left(\bar{\rho}_{L}(x)\right)^{N+2} \Delta Q_{\varepsilon}\left(\bar{I}_{L}(x)\right)
$$

But

$$
\begin{aligned}
& \Delta Q_{\varepsilon}\left(\bar{I}_{L}(x)\right)=\left(\mathcal{E}_{\bar{\sigma}}(\beta)\right)_{\varepsilon}\left(Q _ { \varepsilon } ( \overline { I } _ { L } ( x ) ) F _ { \delta , \mu } \left(\nabla Q_{\varepsilon}\left(\bar{I}_{L}(x)\right)=\right.\right. \\
& =\left(\mathcal{E}_{\bar{\sigma}}(\beta)\right)_{\varepsilon}\left(\left(1 / \rho_{L}(x)\right)^{N-2} \vartheta_{\varepsilon}^{L}(x)\right) F_{\delta, \mu}\left(\nabla \vartheta_{\varepsilon}^{L}(x)+A_{L}^{\varepsilon}(x)\right)
\end{aligned}
$$

where

$$
\begin{gathered}
A_{L}^{\varepsilon}(x)=\nabla Q_{\varepsilon}\left(\bar{I}_{L}(x)\right)-\nabla \vartheta_{\varepsilon}^{L}(x)= \\
=\nabla Q_{\varepsilon}\left(\bar{I}_{L}(x)\right)-\nabla\left[\left(\bar{\rho}_{L}(x)\right)^{N-2}\right] Q_{\varepsilon}\left(\bar{I}_{L}(x)\right)-\left(\bar{\rho}_{L}(x)\right)^{N-2} \nabla\left[Q_{\varepsilon}\left(\bar{I}_{L}(x)\right)\right]
\end{gathered}
$$

This way,

$$
\begin{gathered}
\left|\nabla Q_{\varepsilon}\left(\bar{I}_{L}(x)\right)-\left(\bar{\rho}_{L}(x)\right)^{N-2} \nabla\left[Q_{\varepsilon}\left(\bar{I}_{L}(x)\right)\right]\right|= \\
=\sup _{|v|=1}\left\langle\nabla Q_{\varepsilon}\left(\bar{I}_{L}(x)\right)-\left(\bar{\rho}_{L}(x)\right)^{N-2} \nabla\left[Q_{\varepsilon}\left(\bar{I}_{L}(x)\right)\right], v\right\rangle \leq \\
\leq\left|1-\left(\bar{\rho}_{L}(x)\right)^{N-2}\right| \cdot\left|\nabla Q_{\varepsilon}\left(\bar{I}_{L}(x)\right)\right|+ \\
+\left|\bar{\rho}_{L}(x)\right|^{N-2}\left|\nabla Q_{\varepsilon}\left(\bar{I}_{L}(x)\right)\right| \cdot|| I d_{\mathbb{R}^{N}}-D \bar{I}_{L}(x) \|\left.\right|_{\mathcal{L}\left(\mathbb{R}^{N}\right)}
\end{gathered}
$$

This way, since $Q_{\varepsilon}\left(\bar{I}_{L}(x)\right)$ and $\nabla Q_{\varepsilon}\left(\bar{I}_{L}(x)\right)$ are uniformly bounded in $Q_{L_{0}}$ (recall $Q_{\varepsilon}$ are translations of rescalings of $P$ given in Lemma (6.6) by 7.5 and (7.7)

$$
A_{L}^{\varepsilon} \rightarrow 0 \text { uniformly in } Q_{L_{0}} \text { as } L \rightarrow \infty \text { uniformly in } \varepsilon
$$

Since $F$ is Lipschitz continuous, we have for $x \in Q_{L_{0}}$ and $L$ large enough

$$
\begin{gathered}
F\left(\nabla \vartheta_{\varepsilon}^{L}(x)+A_{L}^{\varepsilon}\right)+\mu \geq F\left(\nabla \vartheta_{\varepsilon}^{L}(x)+A_{L}^{\varepsilon}\right)+\operatorname{Lip}(F)\left|A_{L}^{\varepsilon}\right| \geq F\left(\nabla \vartheta_{\varepsilon}^{L}(x)\right) \\
(1+\delta)\left(\bar{\rho}_{L}(x)\right)^{N-2} \geq 1+\frac{\delta}{2}
\end{gathered}
$$


Also, by Lemma 6.2 , since $\bar{\sigma}>\sigma>1$

$$
\left(\mathcal{E}_{\bar{\sigma}}(\beta)\right)_{\varepsilon}\left(\left(1 / \rho_{L}(x)\right)^{N-2} \vartheta_{\varepsilon}^{L}(x)\right) \geq\left(\mathcal{E}_{\sigma}(\beta)\right)_{\varepsilon}\left(\vartheta_{\varepsilon}^{L}(x)\right)
$$

Combining the estimates above, we conclude that choosing $L$ large enough, we have for $x \in Q_{L_{0}}$ uniformly in $\varepsilon$

$$
\begin{aligned}
\Delta \vartheta_{\varepsilon}^{L}(x) & \geq\left(1+\frac{\delta}{2}\right)\left(\mathcal{E}_{\sigma}(\beta)\right)_{\varepsilon}\left(\vartheta_{\varepsilon}^{L}(x)\right) F\left(\nabla \vartheta_{\varepsilon}^{L}(x)\right) \geq \\
& \geq\left(\mathcal{E}_{\sigma}(\beta)\right)_{\varepsilon}\left(\vartheta_{\varepsilon}^{L}(x)\right) F\left(\nabla \vartheta_{\varepsilon}^{L}(x)\right)
\end{aligned}
$$

It follows from the proof of Lemma (6.6)a) that there exists $\bar{\gamma}>\gamma$ such that

$$
\bar{\gamma}<\left(\bar{Q}_{\varepsilon}\right)_{s}<\bar{\alpha} \text { with } \bar{Q}_{\varepsilon}(0)=0
$$

We can easily check that the following properties below hold

(1) $\bar{Q}_{\varepsilon}(s) \leq \bar{\gamma} s$ for $s \in(-\infty, 0]$ and $\bar{Q}_{\varepsilon}(s) \leq \bar{\alpha} s$ for $s \in[0, \infty)$;

(2) $x \in Q_{L_{0}} \Rightarrow\left(\bar{I}_{L}(x)\right)_{1} \leq-L+\frac{L^{2}}{L-x_{1}}=: \tau_{L}\left(x_{1}\right)$ with

$$
\tau_{L} \geq 0 \text { in }\left\{x_{1} \geq 0\right\} \text { and } \tau_{L} \leq 0 \text { in }\left\{x_{1} \leq 0\right\} ;
$$

(3) If $\tau>0$ is a small number, for $L$ large enough, we have

$$
\begin{gathered}
1-\tau \leq \bar{\rho}_{L} \leq 1+\tau \text { in } Q_{L_{0}} \\
1-\tau \leq \frac{d}{d x_{1}} \tau_{L} \leq 1+\tau \text { in }\left[-4 L_{0}, 4 L_{0}\right]
\end{gathered}
$$

Since,

$$
\frac{d}{d x_{1}} \tau_{L}\left(x_{1}\right)=\frac{L^{2}}{\left(L-x_{1}\right)^{2}} \rightarrow 1 \text { uniformly in }\left[-4 L_{0}, 4 L_{0}\right]
$$

From these, it is easy to observe the following estimates

For $x \in\left\{x_{1} \leq 0\right\} \cap Q_{L_{0}}$,

$$
\begin{gathered}
\vartheta_{\varepsilon}^{L}(x)=\left(\bar{\rho}_{L}(x)\right)^{N-2} Q_{\varepsilon}\left(\bar{I}_{L}(x)\right) \leq(1-\tau)^{N-2} \bar{Q}_{\varepsilon}\left(\left(\bar{I}_{L}(x)\right)_{1}\right) \leq \\
\leq(1-\tau)^{N-2} \bar{Q}_{\varepsilon}\left(\tau_{L}\left(x_{1}\right)\right) \leq
\end{gathered}
$$




$$
\leq(1-\tau)^{N-1} \bar{\gamma} x_{1}=-\widetilde{\gamma} x_{1}^{-}
$$

Similarly, for $x \in\left\{x_{1} \geq 0\right\} \cap Q_{L_{0}}$

$$
\begin{gathered}
\vartheta_{\varepsilon}^{L}(x)=\left(\bar{\rho}_{L}(x)\right)^{N-2} \bar{Q}_{\varepsilon}\left(\left(\bar{I}_{L}(x)\right)_{1}\right) \leq(1+\tau)^{N-2} \bar{Q}_{\varepsilon}\left(\tau_{L}\left(x_{1}\right)\right) \leq \\
(1+\tau)^{N-1} \bar{\alpha} x_{1}=\widetilde{\alpha} x_{1}^{+}
\end{gathered}
$$

We can use also, similar ideas, to obtain estimates along the boundary. In this case, the estimates will be 1-dimensional. Indeed, if $x \in Q_{L_{0}} \cap$ $\left\{x=\left(x_{1}, x^{\prime}\right) \in \mathbb{R}^{N} ;\left|x^{\prime}\right|=L_{0}\right\}$ then

$$
\vartheta_{\varepsilon}(x)=\left(\widetilde{\rho}_{L}\left(x_{1}\right)\right)^{N-2} Q_{\varepsilon}\left(\bar{I}_{L}(x)\right)=\left(\widetilde{\rho}_{L}\left(x_{1}\right)\right)^{N-2} \bar{Q}_{\varepsilon}\left(\varphi_{L}\left(x_{1}\right)\right)
$$

where

$$
\widetilde{\rho}_{L}\left(x_{1}\right)=\frac{L}{\sqrt{\left(L-x_{1}\right)^{2}+L_{0}^{2}}}
$$

and

$$
\varphi_{L}\left(x_{1}\right):=\left(\widetilde{I}_{L}(x)\right)_{1}=-L+\frac{L^{2}}{\left(L-x_{1}\right)^{2}+L_{0}^{2}}\left(L-x_{1}\right)
$$

Now, let us observe that $\varphi_{L}$ has the following properties,

$$
\begin{gathered}
\frac{d \varphi_{L}}{d x_{1}}\left(x_{1}\right)=\frac{L^{2}\left(\left(L-x_{1}\right)^{2}-L_{0}^{2}\right)}{\left[\left(L-x_{1}\right)^{2}+L_{0}^{2}\right]^{2}} \rightarrow 1 \text { uniformly in }\left[-4 L_{0}, 4 L_{0}\right] \\
\varphi_{L}\left(x_{1}\right)=0 \Longleftrightarrow x_{1}=d:=\frac{L-\sqrt{L^{2}-4 L_{0}^{2}}}{2}>0 \\
\varphi_{L} \geq 0 \text { in }\left[-4 L_{0}, d\right], \quad \varphi_{L} \leq \text { in }\left[d, 4 L_{0}\right]
\end{gathered}
$$

Also,

$$
\left\{\vartheta_{\varepsilon}^{L}(x)=0\right\} \cap Q_{L_{0}} \cap\left\{x \in Q_{L_{0}} ;\left|x^{\prime}\right|=L_{0}\right\} \Longleftrightarrow x_{1}=d
$$

(using that $g(x)=\sqrt{x}$ is Lipschitz away from the origin, we can easily estimate $d<\frac{L_{0}}{10}$ ). If $\tau>0$ is a small enough, again for $L$ large enough,

$$
1-\tau \leq \frac{d \varphi_{L}}{d x_{1}}\left(x_{1}\right) \leq 1+\tau \text { for } x_{1} \in\left[-4 L_{0}, 4 L_{0}\right]
$$


and

$$
1-\tau \leq \widetilde{\rho}_{L}\left(x_{1}\right) \leq 1+\tau \text { for } x_{1} \in\left[-4 L_{0}, 4 L_{0}\right]
$$

This way, we have for $x \in Q_{L_{0}} \cap\left\{x=\left(x_{1}, x^{\prime}\right) \in \mathbb{R}^{N} ;\left|x^{\prime}\right|=L_{0}\right\} \cap\left\{x_{1} \geq d\right\}$

$$
\begin{gathered}
\vartheta_{\varepsilon}^{L}(x) \leq(1+\tau)^{N-2} \bar{Q}_{\varepsilon}\left(\varphi_{L}\left(x_{1}\right)\right) \leq(1+\tau)^{N-2} \bar{\alpha} \varphi_{L}\left(x_{1}\right) \leq \\
\leq(1+\tau)^{N-1} \bar{\alpha}\left(x_{1}-d\right)=\widetilde{\alpha}\left(x_{1}-d\right)^{+}
\end{gathered}
$$

Similarly,

$$
\begin{aligned}
x & \in Q_{L_{0}} \cap\left\{x=\left(x_{1}, x^{\prime}\right) \in \mathbb{R}^{N} ;\left|x^{\prime}\right|=L_{0}\right\} \cap\left\{x_{1} \leq d\right\} \Rightarrow \\
& \Rightarrow \vartheta_{\varepsilon}^{L}(x) \leq-(1-\tau)^{N-1} \bar{\gamma}\left(d-x_{1}\right)=-\widetilde{\gamma}\left(x_{1}-d\right)^{-}
\end{aligned}
$$

The fact that $Q_{\varepsilon} \leq \vartheta_{\varepsilon}^{L}$ along span $\left\{e_{1}\right\}$ is straightforward. If we choose now $\bar{L}$ large enough in such way that all the estimates above holds, we define for every $\varepsilon$

$$
\vartheta_{\varepsilon}:=\vartheta_{\varepsilon}^{\bar{L}}
$$

Thus, $a$ ) and $c$ ) are proven. b) follows from the geometric properties of inversions.

Proposition 7.3 (Below condition barrier - I). $0<\bar{\sigma}<\sigma<1, \delta, \mu<0$ and $\bar{\alpha}, \gamma>0$ be such that

$$
0<H_{\delta, \mu}(\bar{\alpha})-M_{\bar{\sigma}}<H_{\delta, \mu}(\gamma)
$$

Let $0<\alpha^{\star}<\bar{\alpha}$ be close to $\bar{\alpha}$. There exists a function $\chi_{\varepsilon} \in C^{2}\left(Q_{L_{0}}\right)$ such that for every $\varepsilon>0$

a) $\Delta \chi_{\varepsilon}(x) \leq \beta_{\varepsilon}\left(\chi_{\varepsilon}(x)\right) F\left(\nabla \chi_{\varepsilon}(x)\right)$ for $x$ in $Q_{L_{0}}$;

b) one has

$$
\begin{gathered}
\chi_{\varepsilon}>0 \text { in } Q_{L_{0}} \cap \mathbb{B}_{\star}{ }^{C} \\
\chi_{\varepsilon}<0 \text { in } Q_{L_{0}} \cap \mathbb{B}_{\star}^{\circ} \\
\chi_{\varepsilon}=0 \text { on } Q_{L_{0}} \cap \mathbb{S}_{\star} \\
\mathbb{S}_{\star} \cap \partial Q_{L_{0}} \subset\left\{x_{1}=d_{\star}\right\}, \quad d_{\star}=d_{\star}\left(\text { radius of } \mathbb{S}_{\star}, L_{0}\right)>0
\end{gathered}
$$

where $\mathbb{S}_{\star}=\partial \mathbb{B}_{\star}$, and $\mathbb{B}_{\star}$ is a closed ball completely contained in the half space $\left\{x_{1} \leq 0\right\}$, centered in the negative semi-axis generated by $e_{1}$ and tangent to the hyperplane $\left\{x_{1}=0\right\}$; 
c) There exist $0<\widetilde{\alpha}<\alpha^{\star}$ and $0<\widetilde{\gamma}<\gamma$ and constants $C, D>0$ not depending on $\varepsilon$ such that if $\mathcal{W}^{\star}(x)=\widetilde{\alpha} x_{1}^{+}-\widetilde{\gamma} x_{1}^{-}$, then

$$
\mathcal{W}_{\varepsilon}^{\star}(x):=\mathcal{W}^{\star}\left(x-\varepsilon D e_{1}\right)+C \varepsilon \leq \chi_{\varepsilon}(x) \text { for all } x \in Q_{L_{0}}
$$

$\mathcal{W}^{\star}\left(x+\left(d_{\star}-\varepsilon D\right) e_{1}\right) \leq \chi_{\varepsilon}(x)$ for $x \in Q_{L_{0}} \cap\left\{x=\left(x_{1}, x^{\prime}\right) \in \mathbb{R}^{N} ;\left|x^{\prime}\right|=L_{0}\right\}$

$$
Q_{\varepsilon} \geq \chi_{\varepsilon} \text { along } \operatorname{span}\left\{e_{1}\right\}
$$

where $Q_{\varepsilon}(x):=\bar{Q}_{\varepsilon}\left(x_{1}\right)$, and $\bar{Q}_{\varepsilon}(s)=P_{\varepsilon}\left(s+a_{\varepsilon}\right), P_{\varepsilon}$ is the solution $\left(\mathcal{E}_{\bar{\alpha}, \delta, \mu, \sigma}^{\varepsilon}\right)$ where $a_{\varepsilon}$ is chosen such that $\bar{Q}_{\varepsilon}(0)=0$. Moreover, $\widetilde{\alpha}$ can be taken as close as we wish from $\alpha^{\star}$.

Proof. The proof is very similar to the proof of Proposition (7.2). As suggested in $c$ ), if we define

$$
Q_{\varepsilon}(x):=\bar{Q}_{\varepsilon}\left(x_{1}\right)
$$

and for $\bar{J}_{L}=J_{L} \circ \mathcal{R}_{1}$ and $\bar{\varrho}_{L}=\varrho \circ \mathcal{R}_{1}$ we set

$$
\chi_{\varepsilon}^{L}(x):=\left(T_{L}\left[Q_{\varepsilon}\right] \circ \mathcal{R}_{1}\right)(x)=T_{L}\left[Q_{\varepsilon}\right]\left(\mathcal{R}_{1}(x)\right)=\left(\bar{\varrho}_{L}(x)\right)^{N-2} Q_{\varepsilon}\left(\bar{J}_{L}(x)\right)
$$

with

$$
\begin{gathered}
\Delta \chi_{\varepsilon}^{L}(x)=\left(\Delta T_{L}\left[Q_{\varepsilon}\right] \circ \mathcal{R}_{1}\right)(x)=\Delta T_{L}\left[Q_{\varepsilon}\right]\left(\mathcal{R}_{1}(x)\right)= \\
=\left(\bar{\varrho}_{L}(x)\right)^{N+2} \Delta Q_{\varepsilon}\left(\bar{J}_{L}(x)\right)= \\
=\left(\bar{\varrho}_{L}(x)\right)^{N+2}\left(\mathcal{E}_{\bar{\sigma}}(\beta)\right)_{\varepsilon}\left(\left(1 / \varrho_{L}(x)\right)^{N-2} \chi_{\varepsilon}^{L}(x)\right) F_{\delta, \mu}\left(\nabla \chi_{\varepsilon}^{L}(x)+\bar{A}_{L}^{\varepsilon}(x)\right)
\end{gathered}
$$

where,

$$
\bar{A}_{L}^{\varepsilon}(x)=\nabla Q_{\varepsilon}\left(\bar{J}_{L}(x)\right)-\nabla \chi_{\varepsilon}^{L}(x)
$$

Proceeding as in the proof of Proposition (7.2), we obtain

$$
\bar{A}_{L}^{\varepsilon} \rightarrow 0 \text { uniformly in } Q_{L_{0}} \text { as } L \rightarrow \infty \text { uniformly in } \varepsilon
$$


Since $\delta, \mu<0$, for $x \in Q_{L_{0}}$ and $L>20 L_{0}$ large enough

$$
(1+\delta)\left(\bar{\varrho}_{L}(x)\right)^{N-2} \leq 1+\frac{\delta}{2}<1
$$

by Lemma 6.2 , if $\bar{\sigma}<\sigma<1$,

$$
\left(\mathcal{E}_{\bar{\sigma}}(\beta)\right)_{\varepsilon}\left(\left(1 / \varrho_{L}(x)\right)^{N-2} \chi_{\varepsilon}^{L}(x)\right) \leq\left(\mathcal{E}_{\sigma}(\beta)\right)_{\varepsilon}\left(\chi_{\varepsilon}^{L}(x)\right)
$$

and thus for $L$ large enough and for $x \in Q_{L_{0}}$,

$$
\begin{gathered}
\Delta \chi_{\varepsilon}^{L}(x) \leq\left(1+\frac{\delta}{2}\right)\left(\mathcal{E}_{\sigma}(\beta)\right)_{\varepsilon}\left(\chi_{\varepsilon}^{L}(x)\right) F\left(\nabla \chi_{\varepsilon}^{L}(x)\right) \leq \\
\left(\mathcal{E}_{\sigma}(\beta)\right)_{\varepsilon}\left(\chi_{\varepsilon}^{L}(x)\right) F\left(\nabla \chi_{\varepsilon}^{L}(x)\right) \leq \beta_{\varepsilon}\left(\chi_{\varepsilon}^{L}(x)\right) F\left(\nabla \chi_{\varepsilon}^{L}(x)\right)
\end{gathered}
$$

Analogously to the proof of Proposition (7.2), by Lemma (6.6)b.1), there exists $0<\bar{\gamma}<\gamma$ such that

$$
\bar{\gamma} \leq\left(\bar{Q}_{\varepsilon}\right)_{s} \leq \bar{\alpha}
$$

It is easy to check properties below

1) There exists a constant $\bar{D}$ such that

$$
\left\{\begin{array}{cl}
\bar{Q}_{\varepsilon}(s) \geq \alpha^{\star} s, & \text { for } s \geq \bar{D} \varepsilon \\
\bar{Q}_{\varepsilon}(s)=\bar{\gamma} s, & \text { for } s \leq 0 \\
\bar{Q}_{\varepsilon}(s) \geq \bar{\gamma} s, & \text { for every } s
\end{array}\right.
$$

2) $x \in Q_{L_{0}} \Rightarrow\left(J_{L}(x)\right)_{1} \geq L-\frac{L^{2}}{L+x_{1}}:=\tau_{L}^{\star}\left(x_{1}\right)$, with

$$
\tau_{L}^{\star} \geq 0 \text { in }\left\{x_{1} \geq 0\right\} \quad \text { and } \quad \tau_{L}^{\star} \leq 0 \text { in }\left\{x_{1} \leq 0\right\}
$$

3) If $\tau>0$ is a small number, for $L$ large enough we have

$$
\begin{gathered}
1-\tau \leq \bar{\varrho}_{L} \leq 1+\tau \text { in } Q_{L_{0}} \\
1-\tau \leq \frac{d}{d x_{1}} \tau_{L}^{\star} \leq 1+\tau \text { in }\left[-4 L_{0}, 4 L_{0}\right]
\end{gathered}
$$


From (3), there exists $D$ such that $x_{1} \geq D \varepsilon \Rightarrow \tau_{L}^{\star}\left(x_{1}\right) \geq \bar{D} \varepsilon$, and thus,

$$
\begin{gathered}
\left\{x_{1} \geq \bar{D} \varepsilon\right\} \cap Q_{L_{0}} \Rightarrow \chi_{\varepsilon}^{L}(x)=\left(\varrho_{L}(x)\right)^{N-2} \bar{Q}_{\varepsilon}\left(\left(\bar{J}_{L}(x)\right)_{1}\right) \geq \\
\geq(1-\tau)^{N-2} \bar{Q}_{\varepsilon}\left(\tau_{L}^{\star}\left(x_{1}\right)\right) \geq(1-\tau)^{N-1} \alpha^{\star} x_{1}
\end{gathered}
$$

Proceeding similarly, we find

$$
\begin{gathered}
x_{1} \leq 0 \Rightarrow \chi_{\varepsilon}^{L}(x) \geq-(1+\tau)^{N-1} \bar{\gamma} x_{1}^{-}=-\widetilde{\gamma} x_{1}^{-} \\
0 \leq x_{1} \leq \bar{D} \varepsilon \Rightarrow \chi_{\varepsilon}^{L}(x) \geq(1-\tau)^{N-1} \bar{\gamma} x_{1}=\gamma^{\star} x_{1}^{+}
\end{gathered}
$$

Setting $C=\gamma^{\star} D$, it follows that

$$
\mathcal{W}_{\varepsilon}^{\star}(x)=\mathcal{W}^{\star}(x-D \varepsilon)+C \varepsilon \leq \chi_{\varepsilon}(x) \text { for all } x \in Q_{L_{0}}
$$

Following the ideas above and proceeding as in the proof of Proposition (7.2), we finish the proof.

Proposition 7.4 (Below condition barrier - II). Let $0<\sigma<\bar{\sigma}<1$ and $\delta, \mu<0$ with $\bar{\alpha}>0$ such that

$$
H_{\delta, \mu}(\bar{\alpha})<M_{\bar{\sigma}}
$$

Then, there exist a function $\chi_{\varepsilon} \in C^{2}\left(Q_{L_{0}}\right)$ and constants $C, D>0$ (independent of $\varepsilon$ ) satisfying for every $\varepsilon$

a) $\Delta \chi_{\varepsilon}(x) \leq \beta_{\varepsilon}\left(\chi_{\varepsilon}(x)\right) F\left(\nabla \chi_{\varepsilon}(x)\right)$ for $x$ in $Q_{L_{0}}$;

b) $\chi_{\varepsilon} \geq C \varepsilon$ in $Q_{L_{0}}$ and $\chi_{\varepsilon} \leq Q_{\varepsilon}$ for $\left\{x_{1} \geq 0\right\}$; where $Q_{\varepsilon}(x):=P_{\varepsilon}\left(x_{1}\right), P_{\varepsilon}$ solution to $\left(\mathcal{E}_{\bar{\alpha}, \delta, \mu, \sigma}^{\varepsilon}\right)$

c) There exists $0<\widetilde{\alpha}<\bar{\alpha}$ and a constant $C>0$ independent of $\varepsilon$ such that

$$
\chi_{\varepsilon} \geq \widetilde{\alpha} x_{1}^{+}+D \varepsilon \text { for } x \in Q_{L_{0}} \cap\left\{x_{1} \geq 0\right\}
$$

Moreover, $\widetilde{\alpha}$ can be taken as close as we wish from $\bar{\alpha}$. 
d) There exists a negative number $d_{\star}$ independent of $\varepsilon$ such that on

$$
\begin{aligned}
& \chi_{\varepsilon}(x) \rightarrow g\left(x_{1}\right) \text { uniformly on }\left\{x=\left(x_{1}, x^{\prime}\right) \in Q_{L_{0}} ;\left|x^{\prime}\right|=L_{0}\right\} \\
& \text { and } \\
& \qquad g\left(x_{1}\right) \geq \widetilde{\alpha}\left(x_{1}-d_{\star}\right) \quad \text { for } x_{1} \geq d_{\star}
\end{aligned}
$$

Proof. Defining $\chi_{\varepsilon}^{L}(x)=\left(T_{L}\left[Q_{\varepsilon}\right] \circ \mathcal{R}_{1}\right)(x)$ as in Proposition $(7.3)$, where $Q_{\varepsilon}(x)$ is specified above, then, for $L$ large enough,

$$
\Delta \chi_{\varepsilon}^{L}(x) \leq \beta_{\varepsilon}\left(\chi_{\varepsilon}(x)\right) F\left(\nabla \chi_{\varepsilon}(x)\right) \text { for } x \text { in } Q_{L_{0}}
$$

But now, by Lemma (6.6)b.2), we have for $C=(1-\tau)^{N-2} \bar{\kappa}_{\bar{\sigma}}$

$$
\chi_{\varepsilon}^{L}(x)=\left(\varrho_{L}(x)\right)^{N-2} \bar{Q}_{\varepsilon}\left(\left(\bar{J}_{L}(x)\right)_{1}\right) \geq C \varepsilon \quad \forall x \in Q_{L_{0}}
$$

and also, for $D=(1-\tau)^{N-2} \kappa_{\bar{\sigma}}^{+}$and $x \in Q_{L_{0}} \cap\left\{x_{1} \geq 0\right\}$

$$
\chi_{\varepsilon}^{L}(x) \geq(1-\tau)^{N-2} \bar{Q}_{\varepsilon}\left(\tau_{L}^{\star}\left(x_{1}\right)\right) \geq(1-\tau)^{N-1} \alpha x_{1}^{+}+D \varepsilon=\widetilde{\alpha} x_{1}^{+}+D \varepsilon
$$

Now, as before, we fix a universal $L$ for which the estimates above hold uniformly in $\varepsilon$. From, Lemma (6.6) b.2), we conclude that for some $\bar{\gamma}>0$

$$
Q_{\varepsilon} \rightarrow P^{\star}(x):=\bar{\alpha} x_{1}^{+}+\bar{\gamma} x_{1}^{-} \text {uniformly in } \mathbb{R}^{N}
$$

Since, Kelvin Transforms preserve uniform convergence, we have

$$
\chi_{\varepsilon} \rightarrow T_{L}\left[P^{\star}\right] \circ \mathcal{R}_{1} \text { uniformly in } Q_{L_{0}} \text { as } \varepsilon \rightarrow 0
$$

In particular, for $x \in Q_{L_{0}} \cap\left\{x=\left(x_{1}, x^{\prime}\right) \in \mathbb{R}^{N} ;\left|x^{\prime}\right|=L_{0}\right\}$

$$
\chi_{\varepsilon} \rightarrow g\left(x_{1}\right):=\left(\widetilde{\varrho}_{L}\left(x_{1}\right)\right)^{N-2} P^{\star}\left(\varphi_{L}^{\star}\left(x_{1}\right)\right) \text { uniformly as } \varepsilon \rightarrow 0
$$

where,

$$
\widetilde{\varrho}_{L}\left(x_{1}\right)=\frac{L}{\sqrt{\left(L+x_{1}\right)^{2}+L_{0}^{2}}}
$$

and 


$$
\varphi_{L}^{\star}\left(x_{1}\right)=L-\frac{L^{2}}{\left(L+x_{1}\right)^{2}+L_{0}^{2}}\left(L+x_{1}\right)
$$

Clearly,

$x_{1} \in\left[-4 L_{0}, 4 L_{0}\right]$ with $g\left(x_{1}\right)=0 \Longleftrightarrow x_{1}=d_{\star}:=\frac{1}{2}\left(-L+\sqrt{L^{2}-4 L_{0}^{2}}\right)<0$

$L$ can be taken large enough such that if $\tau$ is a small number

$$
\frac{d}{d x_{1}} \varphi_{L}^{\star}\left(x_{1}\right) \geq 1-\tau \quad \forall x \in Q_{L_{0}}
$$

and thus,

$$
\varphi_{L}^{\star}\left(x_{1}\right) \geq(1-\tau)\left(x_{1}-d_{\star}\right) \quad \forall x \in Q_{L_{0}}
$$

So,

$$
x_{1} \geq d_{\star} \Rightarrow g\left(x_{1}\right) \geq(1-\tau)^{N-1} \bar{\alpha}\left(x_{1}-d_{\star}\right)=\widetilde{\alpha}\left(x_{1}-d_{\star}\right)
$$

From the convergence, $(7.23), d)$ follows, finishing the proof.

\section{Classification of Global Profiles}

The purpose of this section is to classify the global profiles (2-plane functions) that will appear in the blow-up analysis. The precise statement of the result is the following

Theorem 8.1 (Classification of Global Profiles). Let $v_{\varepsilon_{j}}$ be a family of least viscosity solutions to $(S E)_{\varepsilon_{j}}$ in a domain $\Omega_{j} \subset \mathbb{R}^{N}$ such that $\Omega_{j} \subset \Omega_{j+1}$ and $\cup_{j=1}^{\infty} \Omega_{j}=\mathbb{R}^{N}$. Suppose $v_{\varepsilon_{j}}$ converge to $v(x)$ uniformly on compact subsets of $\mathbb{R}^{N}$. Then we have,

$$
\begin{gathered}
v(x)=\alpha x_{1}^{+}-\gamma x_{1}^{-} \text {with } \alpha>0, \gamma \geq 0 \Longrightarrow H(\alpha)-H(\gamma)=M \\
v(x)=\alpha x_{1}^{+}+\gamma x_{1}^{+} \text {with } \alpha>0, \gamma \geq 0 \Longrightarrow H(\alpha) \leq M
\end{gathered}
$$

Heuristically, the idea of the proof is the following: If the slopes of the limit would satisfy $H(\alpha)-H(\gamma)>M$, then we could use the above condition barriers to construct $\varepsilon$ - regularized 2-plane function which are "almost" strict 
subsolution to $\left(S E_{\varepsilon}\right)$ with uniformly curved free boundary and bigger opening $v$. If we bring them from the right starting at "infinity", this geometry would force a interior contact with $v_{\varepsilon}$ violating Lemma (6.3). Analogously, if $H(\alpha)-H(\gamma)<M$, then we could use a below condition barriers to construct $\varepsilon$ - regularized 2-plane functions with uniformly curved free boundary and smaller opening. If we bring them from the left, starting at infinity, this geometry would force a interior crossing of the graph of $v_{\varepsilon}$ violating the least supersolution conditon. The proof will be divided in several propositions, analyzing different possibilities.

Proposition 8.2. Let $v_{\varepsilon_{j}}$ be viscosity solutions to $(S E)_{\varepsilon_{j}}$ in a domain $\Omega_{j} \subset$ $\mathbb{R}^{N}$ such that $\Omega_{j} \subset \Omega_{j+1}$ and $\cup_{j=1}^{\infty} \Omega_{j}=\mathbb{R}^{N}$. Suppose $v_{\varepsilon_{j}}$ converge to $v=$ $\alpha x_{1}^{+}-\gamma x_{1}^{-}$uniformly on compact subsets of $\mathbb{R}^{N}$, with $\alpha>0, \gamma \geq 0$ and $\varepsilon_{j} \rightarrow 0$. Then,

$$
H(\alpha)-H(\gamma) \leq M
$$

Proof. Let us suppose by contradiction that,

$$
H_{0,0}(\alpha)-H_{0,0}(\gamma)=H(\alpha)-H(\gamma)>M
$$

This way, we can find $0<\bar{\alpha}<\alpha$ and $\bar{\sigma}>1$ such that $H_{0,0}(\bar{\alpha})-H_{0,0}(\gamma)>$ $M_{\sigma}=\bar{\sigma}^{2} M>M$. So, by continuity, there exist $\delta>0, \mu>0$ such that

$$
H_{\delta, \mu}(\bar{\alpha})-H_{\delta, \mu}(\gamma)>M_{\bar{\sigma}}
$$

Thus, we are in conditions to use the above condition barriers constructed in Proposition 7.2 with $\alpha>\widetilde{\alpha}$. In what follows, we will freely use them as well as the notation employed there. Let $\eta>0$ small be given. By assumption, we can find $\varepsilon_{0}=\varepsilon_{0}(\eta)>0$ such that

$$
\varepsilon<\varepsilon_{0} \Longrightarrow\left\|v_{\varepsilon}-v\right\|_{L^{\infty}\left(Q_{L_{0}}\right)}<\eta
$$

Setting $c_{1}=\frac{1}{\widetilde{\gamma}}$ and $c_{2}=c_{1}+\frac{1}{\bar{\gamma}}$, we may assume that $\eta$ is so small that

$$
\left(c_{1}+c_{2}\right) \eta<\frac{d}{4}<\frac{L_{0}}{4}
$$


Setting $Q_{0}=\frac{1}{2} Q_{L_{0}}$ and $Q_{00}=\frac{1}{4} Q_{L_{0}}$, we have that for every $\xi \in \mathbb{R}^{N}$ with $|\xi| \leq L_{0}$, the functions $\left(\vartheta_{\varepsilon}\right)_{\xi}: Q_{0} \rightarrow \mathbb{R}$ given by $\left(\vartheta_{\varepsilon}\right)_{\xi}(x)=\vartheta_{\varepsilon}(x+\xi)$ are well defined. In particular, we can define $\vartheta_{\varepsilon}^{\star}: Q_{0} \rightarrow \mathbb{R}$, given by

$$
\vartheta_{\varepsilon}^{\star}(x)=\vartheta_{\varepsilon}\left(x-c_{1} \eta e_{1}\right)
$$

It is easy to see that

$$
\vartheta_{\varepsilon}^{\star}(x) \leq \mathcal{W}\left(x-c_{1} \eta e_{1}\right)<v(x)-\eta<v_{\varepsilon}(x) \text { for } x \in Q_{00}
$$

If $|T| \leq \frac{L_{0}}{4}$ then we can define $\left(\vartheta_{\varepsilon}^{\star}\right)_{T}: Q_{00} \rightarrow \mathbb{R}$ by

$$
\left(\vartheta_{\varepsilon}^{\star}\right)_{T}(x):=\vartheta_{\varepsilon}^{\star}\left(x+T e_{1}\right)
$$

So, let us consider the set

$$
\Gamma_{\varepsilon}=\left\{0<T \leq \frac{L_{0}}{4} ;\left(\vartheta_{\varepsilon}^{\star}\right)_{T} \leq v_{\varepsilon} \text { in } Q_{00}\right\} \quad \text { and } \mathcal{T}_{\varepsilon}=\sup \Gamma_{\varepsilon}
$$

Let us recall that $Q_{\varepsilon}(x)=\bar{Q}_{\varepsilon}\left(x_{1}\right) \geq \bar{\gamma} x_{1}$ for $x_{1} \geq 0$ and $\vartheta_{\varepsilon} \geq Q_{\varepsilon}$ along $Z=\operatorname{span}\left\{e_{1}\right\}$. In particular, considering $x=l e_{1}$, with $|l| \leq \frac{L_{0}}{4}$ and $l \geq-\frac{\eta}{\bar{\gamma}}$

$$
\vartheta_{\varepsilon}\left(\left(l+\frac{\eta}{\bar{\gamma}}\right) e_{1}\right) \geq \bar{\gamma} l+\eta
$$

but,

$$
\left.\vartheta_{\varepsilon}\left(\left(l+\frac{\eta}{\bar{\gamma}}\right) e_{1}\right)=\vartheta_{\varepsilon}\left(\left(l-c_{1} \eta+c_{2} \eta\right) e_{1}\right)\right)=\left(\vartheta_{\varepsilon}^{\star}\right)_{c_{2} \eta}\left(l e_{1}\right)
$$

Taking now, $l=0$, we find

$$
\left(\vartheta_{\varepsilon}^{\star}\right)_{c_{2} \eta}(0) \geq \eta>v_{\varepsilon}(0)
$$

In other words, if we translate $\vartheta_{\varepsilon}^{\star}$ by $c_{2} \eta$, we have gone to far in terms of touching $v_{\varepsilon}$ by below. This implies that

$$
\mathcal{T}_{\varepsilon} \leq c_{2} \eta
$$

Moreover, there exists $x_{n} \in Q_{00}$ such that for all $n \geq 1$

$$
\left(\vartheta_{\varepsilon}^{\star}\right)_{\mathcal{T}_{\varepsilon}+1 / n}\left(x_{n}^{\varepsilon}\right)>v_{\varepsilon}\left(x_{n}\right)
$$

Passing to a subsequence if necessary, we can assume $x_{n}^{\varepsilon} \rightarrow x_{0}^{\varepsilon}$ as $n \rightarrow \infty$ where $x_{0}^{\varepsilon} \in Q_{00}$. Thus we have, 


$$
\begin{gathered}
\left(\vartheta_{\varepsilon}^{\star}\right) \mathcal{T}_{\varepsilon}\left(x_{0}^{\varepsilon}\right)=v_{\varepsilon}\left(x_{0}\right) . \\
\left(\vartheta_{\varepsilon}^{\star}\right)_{\mathcal{T}_{\varepsilon}} \leq v_{\varepsilon} \text { in } Q_{00}
\end{gathered}
$$

Now, since for $x \in Q_{00}$,

$$
v_{\varepsilon}(x)-\left(\vartheta_{\varepsilon}^{\star}\right)_{\mathcal{T}_{\varepsilon}}(x) \geq v(x)-\eta-\left(\vartheta_{\varepsilon}^{\star}\right)_{\mathcal{T}_{\varepsilon}}(x) \geq v(x)-\eta-\mathcal{W}\left(x-c_{1} \eta e_{1}+\mathcal{T}_{\varepsilon} e_{1}\right)
$$

We have

$$
\begin{gathered}
x \in \partial Q_{00} \cap\left\{x_{1}= \pm L_{0}\right\} \Rightarrow v_{\varepsilon}-\left(\vartheta_{\varepsilon}^{\star}\right) \mathcal{I}_{\varepsilon}(x) \geq \\
\min \left\{(\alpha-\widetilde{\alpha}) L_{0}+A(\varepsilon, \eta),(\widetilde{\gamma}-\gamma) L_{0}+B(\varepsilon, \eta)\right\} \geq c_{3}>0
\end{gathered}
$$

if $\eta$ are chosen small enough, since

$$
\begin{aligned}
& A(\varepsilon, \eta)=\left(\widetilde{\alpha} c_{1} \eta-\widetilde{\alpha} \mathcal{T}_{\varepsilon}-\eta\right) \rightarrow 0 \text { as } \eta \rightarrow 0 \\
& B(\varepsilon, \eta)=\left(\widetilde{\gamma} c_{1} \eta-\widetilde{\gamma} \mathcal{T}_{\varepsilon}-\eta\right) \rightarrow 0 \text { as } \eta \rightarrow 0
\end{aligned}
$$

Now, once

$$
\rho>0 \Longrightarrow v(x)-\mathcal{W}\left(x-\rho e_{1}\right) \geq \min \{\alpha, \widetilde{\gamma}\} \rho \forall x \in \mathbb{R}^{N}
$$

we can estimate,

$$
\begin{gathered}
x \in \partial Q_{00} \cap\left\{x=\left(x_{1}, x^{\prime}\right) \in \mathbb{R}^{N} ;\left|x^{\prime}\right|=L_{0}\right\} \Rightarrow \\
\Rightarrow v_{\varepsilon}(x)-\left(\vartheta_{\varepsilon}^{\star}\right)_{\mathcal{T}_{\varepsilon}}(x) \geq v(x)-\eta-\vartheta_{\varepsilon}\left(x-c_{1} \eta e_{1}+\mathcal{T}_{\varepsilon} e_{1}\right) \geq \\
\geq v(x)-\eta-\mathcal{W}\left(x-c_{1} \eta e_{1}+\mathcal{T}_{\varepsilon} e_{1}-d e_{1}\right) \geq \\
\geq \min \{\alpha, \widetilde{\gamma}\}\left(c_{1} \eta-\mathcal{T}_{\varepsilon}+d\right)-\eta \geq \frac{\min \{\alpha, \widetilde{\gamma}\}}{4} d,
\end{gathered}
$$

for $\eta$ small enough, since $c_{1} \eta-\mathcal{T}_{\varepsilon} \rightarrow 0$ as $\eta \rightarrow 0$. In particular, we conclude that if $\eta>0$ is chosen small enough, on the boundary of $Q_{00},\left(\vartheta_{\varepsilon}^{\star}\right)_{\mathcal{T}_{\varepsilon}}$ is striclty below $v_{\varepsilon}$ for $\varepsilon$ small enough. This forces, the contact point $x_{0}^{\varepsilon} \in \operatorname{int}\left(Q_{00}\right)$. Now, from the translation invariance, Remark $(5.5), \bar{\vartheta}_{\varepsilon}=\left(\vartheta_{\varepsilon}^{\star}\right)_{\mathcal{T}_{\varepsilon}}$ satisfies for some $\sigma>1$, 


$$
\Delta \bar{\vartheta}_{\varepsilon}(x) \geq\left(\mathcal{E}_{\sigma}(\beta)\right)_{\varepsilon}\left(\bar{\vartheta}_{\varepsilon}(x)\right) F\left(\nabla \bar{\vartheta}_{\varepsilon}(x)\right) \text { in } Q_{00}
$$

Since $v_{\varepsilon}$ are solutions to $\left(S E_{\varepsilon}\right)$, this contradicts Lemma 6.3). This way,

$$
H(\alpha)-H(\gamma) \leq M
$$

and the Theorem is proven.

Using the same ideas of Theorem $(8.2)$, we can state next corollary. The proof will follow mutatis-mutandis. Details are left to the reader.

Corollary 8.3. Let $v_{\varepsilon_{j}}$ be viscosity solutions to $(S E)_{\varepsilon_{j}}$ in a domain $\Omega_{j} \subset \mathbb{R}^{N}$ such that $\Omega_{j} \subset \Omega_{j+1}$ and $\cup_{j=1}^{\infty} \Omega_{j}=\mathbb{R}^{N}$. Suppose $v_{\varepsilon_{j}}$ converge to $v=\alpha(x-$ $\left.x_{0}\right)_{1}^{+}+\gamma\left(x-x_{0}\right)_{1}^{+}$uniformly on compact subsets of $\mathbb{R}^{N}$, with $\alpha>0, \gamma \geq 0$ and $\varepsilon_{j} \rightarrow 0$. Then,

$$
H(\alpha) \leq M
$$

Now, we study the situation where the limit is a strict 2 -phase case.

Proposition 8.4. Let $v_{\varepsilon_{j}}$ be a family of least viscosity solutions to $(S E)_{\varepsilon_{j}}$ in a domain $\Omega_{j} \subset \mathbb{R}^{N}$ such that $\Omega_{j} \subset \Omega_{j+1}$ and $\cup_{j=1}^{\infty} \Omega_{j}=\mathbb{R}^{N}$. Suppose $v_{\varepsilon_{j}}$ converge to $v=\alpha\left(x-x_{0}\right)_{1}^{+}-\gamma\left(x-x_{0}\right)_{1}^{-}$uniformly on compact subsets of $\mathbb{R}^{N}$, with $\alpha>0, \gamma>0$ and $\varepsilon_{j} \rightarrow 0$. Then,

$$
H(\alpha)-H(\gamma) \geq M
$$

Proof. Let us suppose by contradiction that,

$$
H_{0,0}(\alpha)-H_{0,0}(\gamma)=H(\alpha)-H(\gamma)<M
$$

This way, we can find $0<\bar{\sigma}<1$ such that

$$
H_{0,0}(\alpha)-M_{\bar{\sigma}}<H_{0,0}(\gamma)
$$

Since $\gamma>0$, we can find $\bar{\alpha}>\alpha$ such that

$$
0<H_{0,0}(\bar{\alpha})-M_{\bar{\sigma}}<H_{0,0}(\gamma)
$$

By continuity, there exist $\delta, \mu<0$ such that 


$$
0<H_{\delta, \mu}(\bar{\alpha})-M_{\bar{\sigma}}<H_{\delta, \mu}(\gamma)
$$

Now, let $\alpha<\alpha^{\star}<\bar{\alpha}$. This way, we are in conditions to use the below condition barriers $\chi_{\varepsilon}$ constructed in Proposition (7.3). Let $\eta>0$ small be given. We can find $\varepsilon_{0}=\varepsilon_{0}(\eta)>0$ such that

$$
\varepsilon<\varepsilon_{0} \Rightarrow\left\|v_{\varepsilon}-v\right\|_{L^{\infty}\left(Q_{L_{0}}\right)}<\eta
$$

Let us set $c_{1}:=\max \left\{\frac{1}{\alpha}, \frac{1}{\gamma}\right\}, c_{2}=c_{1}+\frac{1}{\bar{\gamma}}$ and assume $\eta$ is so small that

$$
\left(c_{1}+c_{2}\right) \eta<\frac{d_{\star}}{4}<\frac{L_{0}}{4}
$$

Setting $Q_{0}=\frac{1}{2} Q_{L_{0}}$ and $Q_{00}=\frac{1}{4} Q_{L_{0}}$, we see that if $\xi \in \mathbb{R}^{N}$ with $|\xi| \leq L_{0}$, the functions $\left(\chi_{\varepsilon}\right)_{\xi}: Q_{0} \rightarrow \mathbb{R}$ given by $\left(\chi_{\varepsilon}\right)_{\xi}(x)=\chi_{\varepsilon}(x+\xi)$ are well defined. In particular, taking $\xi=c_{1} \eta e_{1}$, we define

$$
\chi_{\varepsilon}^{\star}(x)=\chi_{\varepsilon}\left(x+c_{1} \eta e_{1}\right)
$$

It is easy to check that

$$
\chi_{\varepsilon}^{\star}(x) \geq \mathcal{W}^{\star}\left(x+c_{1} \eta e_{1}\right)>v(x)+\eta>v_{\varepsilon}(x) \text { for } x \in Q_{00}
$$

For $|T| \leq \frac{L_{0}}{4}$, we can define $\left(\chi_{\varepsilon}^{\star}\right)_{T}: Q_{00} \rightarrow \mathbb{R}$ by

$$
\left(\chi_{\varepsilon}^{\star}\right)_{T}(x):=\chi_{\varepsilon}^{\star}\left(x-T e_{1}\right)
$$

and consider the set

$$
\Gamma_{\varepsilon}=\left\{0 \leq T \leq \frac{L_{0}}{4} ;\left(\chi_{\varepsilon}^{\star}\right)_{T} \geq v_{\varepsilon} \text { in } Q_{00}\right\} \text { and } \mathcal{T}_{\varepsilon}=\sup \Gamma_{\varepsilon}
$$

Let us recall that $Q_{\varepsilon}(x)=\bar{Q}_{\varepsilon}\left(x_{1}\right) \leq \bar{\gamma} x_{1}$ for $x_{1} \leq 0$ and $\chi_{\varepsilon} \leq Q_{\varepsilon}$ along $Z=\operatorname{span}\left\{e_{1}\right\}$. In particular, considering $x=l e_{1}$, with $|l| \leq \frac{L_{0}}{4}$ and $l \leq \frac{\eta}{\bar{\gamma}}$

$$
\chi_{\varepsilon}\left(\left(l-\frac{\eta}{\bar{\gamma}}\right) e_{1}\right) \leq \bar{\gamma} l-\eta
$$

but

$$
\chi_{\varepsilon}\left(\left(l-\frac{\eta}{\bar{\gamma}}\right) e_{1}\right)=\chi_{\varepsilon}\left(\left(l+\eta c_{1}-\eta c_{2}\right) e_{1}\right)=\left(\chi_{\varepsilon}^{\star}\right)_{c_{2} \eta}\left(l e_{1}\right)
$$

Taking now, $l=0$, we find 


$$
\left(\chi_{\varepsilon}^{\star}\right)_{c_{2} \eta}(0) \leq-\eta<v_{\varepsilon}(0)
$$

This means that if we translate $\chi_{\varepsilon}^{\star}$ by $c_{2} \eta$ we have gone too far in terms of touching $v_{\varepsilon}$ by above. In particular,

$$
\mathcal{T}_{\varepsilon} \leq c_{2} \eta
$$

Setting

$$
Z_{\varepsilon}^{\tau}(x):=\left(\chi_{\varepsilon}^{\star}\right)_{\mathcal{T}_{\varepsilon}+\tau}(x), \quad \tau>0
$$

then, we can find $x_{\varepsilon}^{\tau} \in Q_{00}$ such that

$$
Z_{\varepsilon}^{\tau}\left(x_{\varepsilon}^{\tau}\right)<v_{\varepsilon}\left(x_{\varepsilon}^{\tau}\right)
$$

Let us observe that for $x \in Q_{00}$,

$$
\begin{aligned}
& Z_{\varepsilon}^{\tau}(x)-v_{\varepsilon}(x) \geq \mathcal{W}_{\varepsilon}^{\star}\left(x+\left(c_{1} \eta-\mathcal{T}_{\varepsilon}-\tau\right) e_{1}\right)-v(x)-\eta \geq \\
& \geq \mathcal{W}^{\star}\left(\left(x+\left(c_{1} \eta-\mathcal{T}_{\varepsilon}-\tau\right) e_{1}\right)-\varepsilon D e_{1}\right)+C \varepsilon-v(x)-\eta
\end{aligned}
$$

In particular,

$$
\begin{gathered}
x \in Q_{00} \cap\left\{x_{1}= \pm L_{0}\right\} \Rightarrow \\
\Rightarrow Z_{\varepsilon}^{\tau}(x)-v_{\varepsilon}(x) \geq \\
\geq \min \left\{(\widetilde{\alpha}-\alpha) L_{0}+\bar{A}(\eta, \varepsilon, \tau),(\gamma-\widetilde{\gamma}) L_{0}+\bar{B}(\eta, \varepsilon, \tau)\right\} \geq c_{4}>0
\end{gathered}
$$

if $\eta$ and $\tau$ are chosen small enough, since

$$
\begin{aligned}
& \bar{A}(\eta, \varepsilon, \tau)=\widetilde{\alpha}\left(c_{1} \eta-\mathcal{T}_{\varepsilon}-\tau-\varepsilon D\right)+C \varepsilon-\eta \rightarrow 0 \text { as } \varepsilon, \eta, \tau \rightarrow 0 \\
& \bar{B}(\eta, \varepsilon, \tau)=\widetilde{\gamma}\left(c_{1} \eta-\mathcal{T}_{\varepsilon}-\tau-\varepsilon D\right)+C \varepsilon-\eta \rightarrow 0 \text { as } \varepsilon, \eta, \tau \rightarrow 0
\end{aligned}
$$

Furthermore, by (7.16), if $x \in Q_{00} \cap\left\{x=\left(x_{1}, x^{\prime}\right) \in \mathbb{R}^{N} ;\left|x^{\prime}\right|=L_{0}\right\}$

$$
\begin{gathered}
Z_{\varepsilon}^{\tau}(x)-v_{\varepsilon}(x) \geq \mathcal{W}^{\star}\left(x+\left(c_{1} \eta-\mathcal{T}_{\varepsilon}-\tau\right) e_{1}+\left(d_{\star}-\varepsilon D\right) e_{1}\right)-v(x)-\eta \geq \\
\geq \min \{\bar{\alpha}, \gamma\}\left(c_{1} \eta-\mathcal{T}_{\varepsilon}-\tau+d_{\star}-\varepsilon D\right)>c_{5}>0
\end{gathered}
$$


for $\varepsilon, \eta, \tau$ small enough, since $c_{1} \eta-\mathcal{T}_{\varepsilon}-\tau-\varepsilon D \rightarrow 0$ as $\varepsilon, \eta, \tau \rightarrow 0$. This way, by the translation invariance of $\left(S E_{\varepsilon}\right)$, Remark (5.5), $Z_{\varepsilon}^{\tau}$ is a supersolution of $\left(S E_{\varepsilon}\right)$ in $Q_{00}$. Finally, for $\eta, \tau, \varepsilon$ small enough, we have

$$
\begin{gathered}
Z_{\varepsilon}^{\tau} \geq v_{\varepsilon} \text { in } Q_{00} \\
Z_{\varepsilon}^{\tau}\left(x_{\varepsilon}^{\tau}\right)<v_{\varepsilon}\left(x_{\varepsilon}^{\tau}\right) \text { with } x_{\varepsilon}^{\tau} \in \operatorname{int}\left(Q_{00}\right)
\end{gathered}
$$

which contradicts the fact that $v_{\varepsilon}$ is the least supersolution of $\left(S E_{\varepsilon}\right)$. This way, $H(\alpha)-H(\gamma) \geq M$ and the Theorem is proven.

Finally, we treat the case where the profile is of one-phase type.

Proposition 8.5. Let $v_{\varepsilon_{j}}$ be a family of least viscosity solutions to $(S E)_{\varepsilon_{j}}$ in a domain $\Omega_{j} \subset \mathbb{R}^{N}$ such that $\Omega_{j} \subset \Omega_{j+1}$ and $\cup_{j=1}^{\infty} \Omega_{j}=\mathbb{R}^{N}$. Suppose $v_{\varepsilon_{j}}$ converge to $v=\alpha\left(x-x_{0}\right)_{1}^{+}$uniformly on compact subsets of $\mathbb{R}^{N}$, with $\alpha>0$ and $\varepsilon_{j} \rightarrow 0$. Then,

$$
H(\alpha) \geq M
$$

Proof. The proof is similar to the proof of Theorem 8.4. Once more, let us assume by contradiction that $H(\alpha)<M$. As before, we can find $\bar{\alpha}>\alpha$, $\delta, \mu<0$ and $\bar{\sigma}<1$ such that

$$
H_{\delta, \mu}(\bar{\alpha})<M_{\bar{\sigma}}
$$

Let us now choose, $\alpha<\widetilde{\alpha}<\bar{\alpha}$. We are now in conditions to use the barriers constructed in Proposition (7.4). By assumption, there exists $\varepsilon_{0}=\varepsilon_{0}(\eta)$ such that

$$
\varepsilon \leq \varepsilon_{0} \Rightarrow\left\|v_{\varepsilon}-v\right\|_{L^{\infty}\left(Q_{L_{0}}\right)}<\kappa_{2} \eta
$$

Setting $Q_{0}=\frac{1}{2} Q_{L_{0}}$ and $Q_{00}=\frac{1}{4} Q_{L_{0}}$, we see that if $\xi \in \mathbb{R}^{N}$ with $|\xi| \leq L_{0}$, the functions $\left(\chi_{\varepsilon}\right)_{\xi}: Q_{0} \rightarrow \mathbb{R}$ given by $\left(\chi_{\varepsilon}\right)_{\xi}(x)=\chi_{\varepsilon}(x+\xi)$ are well defined.

Let us set

$$
Q_{L_{0}}^{\eta}=\left\{x=\left.\left(x_{1}, x^{\prime}\right) \in \mathbb{R}^{N}|| x\right|_{\infty}=\max \left\{\left|x_{1}\right|,\left|x^{\prime}\right|\right\} \leq 4 L_{0}-2 \eta\right\}
$$

Let us define $c_{1}:=2 \kappa_{2} / \widetilde{\alpha}+3$ and $c_{2}:=c_{1}+2 / \bar{\alpha}$, and consider $\eta$ so small that,

$$
\left(c_{1}+c_{2}\right) \eta<\frac{d_{\star}}{4}<\frac{L_{0}}{4}
$$


Observe, that by the exponential decay in the interior, Lemma (6.5), there exists a constant $C_{\eta}$ such that

$$
x \in Q_{00} \cap\left\{x_{1} \leq-2 \eta\right\} \Rightarrow v_{\varepsilon}^{+} \leq C_{\eta} \varepsilon^{3}
$$

Now, taking the barrier constructed in Proposition (7.4), by b), $\chi_{\varepsilon} \geq C \varepsilon$ in $Q_{L_{0}}$. Let us define

$$
\chi_{\varepsilon}^{\star}(x)=\chi_{\varepsilon}\left(x+c_{1} \eta e_{1}\right)
$$

Then, if for $x_{1} \geq-c_{1} \eta$ we have

$$
\chi_{\varepsilon}^{\star}(x) \geq \widetilde{\alpha}\left(x_{1}+c_{1} \eta e_{1}\right)+D \varepsilon \geq \widetilde{\alpha} x_{1}+2 \kappa_{2} \eta+3 \widetilde{\alpha} \eta+D \varepsilon
$$

In particular, $x_{1} \geq-3 \eta \Rightarrow \chi_{\varepsilon}^{\star}(x)>2 \kappa_{2} \eta$. This way, there exists $\varepsilon_{1}=$ $\varepsilon_{1}(\eta)<\varepsilon_{0}$, we have $\chi_{\varepsilon}-v_{\varepsilon}>0$ in $Q_{0}$ since

$$
\begin{gathered}
\chi_{\varepsilon}^{\star}-v_{\varepsilon} \geq C \varepsilon-C_{\eta} \varepsilon^{3}>0 \quad \text { in } \quad Q_{0} \cap\left\{x_{1} \leq-2 \eta\right\} \\
\chi_{\varepsilon}^{\star}-v_{\varepsilon} \geq \kappa_{2} \eta \quad \text { in } \quad Q_{0} \cap\left\{x_{1} \geq-3 \eta\right\}
\end{gathered}
$$

For $|T| \leq \frac{L_{0}}{4}$, we can define $\left(\chi_{\varepsilon}^{\star}\right)_{T}: Q_{00} \rightarrow \mathbb{R}$ by

$$
\left(\chi_{\varepsilon}^{\star}\right)_{T}(x):=\chi_{\varepsilon}^{\star}\left(x-T e_{1}\right)
$$

and consider the set

$$
\Gamma_{\varepsilon}=\left\{0 \leq T \leq \frac{L_{0}}{4} ;\left(\chi_{\varepsilon}^{\star}\right)_{T} \geq v_{\varepsilon} \text { in } Q_{00}\right\} \text { and } \mathcal{T}_{\varepsilon}=\sup \Gamma_{\varepsilon}
$$

Now, let us recall that

$$
\chi_{\varepsilon}\left(l e_{1}\right) \leq Q_{\varepsilon}\left(l e_{1}\right)=P_{\varepsilon}(l)=\bar{\alpha} l+\varepsilon \kappa_{\bar{\sigma}}^{+} \quad \text { for } \quad l \geq 0
$$

In particular, if $l \geq\left(c_{2}-c_{1}\right) \eta$ and $l \leq \frac{L_{0}}{4}$, then

$$
\left(\chi_{\varepsilon}^{\star}\right)_{c_{2} \eta}\left(l e_{1}\right)=\chi_{\varepsilon}\left(l e_{1}+\left(c_{1}-c_{2}\right) \eta e_{1}\right) \leq \bar{\alpha} l+\bar{\alpha}\left(c_{1}-c_{2}\right) \eta+\varepsilon \kappa_{\bar{\sigma}}^{+}
$$

Taking $l=2 \eta / \bar{\alpha}$, for $\varepsilon$ small enough,

$$
\left(\chi_{\varepsilon}^{\star}\right)_{c_{2} \eta}\left(\frac{2 \eta}{\bar{\alpha}} e_{1}\right)=\chi_{\varepsilon}(0)=\varepsilon \kappa_{\bar{\sigma}}^{+}<\frac{2 \alpha \eta}{\bar{\alpha}}=v\left(\frac{2 \eta}{\bar{\alpha}} e_{1}\right)
$$


In other words, if we translate $\chi_{\varepsilon}^{\star}$ by $c_{2} \eta$ we have gone to far in terms of touching $v_{\varepsilon}$ by above. This implies, that

$$
0 \leq \mathcal{T}_{\varepsilon} \leq c_{2} \eta
$$

Let us we define for $0<\tau<L_{0} / 10$ a small number,

$$
Z_{\varepsilon}^{\tau}(x):=\left(\chi_{\varepsilon}^{\star}\right)_{\mathcal{T}_{\varepsilon}+\tau}(x), \quad \tau>0
$$

Now, we estimate

$$
\begin{gathered}
x \in \partial Q_{00} \cap\left\{x_{1}=L_{0}\right\} \Rightarrow Z_{\varepsilon}^{\tau}(x)-v_{\varepsilon}(x) \geq Z_{\varepsilon}^{\tau}(x)-v(x)-\eta \geq \\
\geq \chi_{\varepsilon}\left(x+c_{1} \eta e_{1}-\mathcal{T}_{\varepsilon} e_{1}-\tau e_{1}\right)-v(x)-\eta \geq \\
\geq(\widetilde{\alpha}-\alpha) L_{0}+\bar{A}(\varepsilon, \eta, \tau) \geq \\
\geq \frac{1}{4}(\widetilde{\alpha}-\alpha) L_{0}
\end{gathered}
$$

since $\bar{A}(\varepsilon, \eta, \tau)=\widetilde{\alpha}\left(c_{1} \eta-c_{2} \eta-\tau\right)+D \varepsilon-\eta \rightarrow 0$ as $\varepsilon, \eta, \tau \rightarrow 0$.

Clearly, for $\eta, \tau, \varepsilon$ small enough,

$$
x \in \partial Q_{00} \cap\left\{x_{1}=-L_{0}\right\} \Rightarrow Z_{\varepsilon}^{\tau}-v_{\varepsilon}>C \varepsilon-C_{\eta} \varepsilon^{3}>0
$$

Finally, let us see that

$$
x \in \partial Q_{00} \cap\left\{x=\left(x_{1}, x^{\prime}\right) \in Q_{L_{0}} ;\left|x^{\prime}\right|=L_{0}\right\} \Rightarrow Z_{\varepsilon}^{\tau}(x)>v_{\varepsilon}(x)
$$

Indeed, choosing $\eta, \tau$ small enough, $d_{\star}-c_{1} \eta+\mathcal{T}_{\varepsilon}+\tau<\frac{3}{4} d_{\star}$. We can assume, passing to a subsquence, if necessary that, $\mathcal{T}_{\varepsilon} \rightarrow \mathcal{T}$ as $\varepsilon \rightarrow 0$. By the convergence given in Proposition (7.4)d),

$$
Z_{\varepsilon}^{\tau} \rightarrow G \quad \text { uniformly in } \quad \partial Q_{00} \cap\left\{x=\left(x_{1}, x^{\prime}\right) \in Q_{L_{0}} ;\left|x^{\prime}\right|=L_{0}\right\}
$$

where,

$$
G\left(x_{1}\right)=g\left(x_{1}+c_{1} \eta-\mathcal{T}-\tau\right)
$$

Additionally, if $x_{1} \geq d_{\star}-c_{1} \eta+\mathcal{T}+\tau$, then

$$
G\left(x_{1}\right) \geq \widetilde{\alpha}\left(x_{1}-d_{\star}+c_{1} \eta-\mathcal{T}-\tau\right)
$$


So, for $\varepsilon$ small enough and $x \in \partial Q_{00} \cap\left\{x=\left(x_{1}, x^{\prime}\right) \in Q_{L_{0}} ;\left|x^{\prime}\right|=L_{0}\right\} \cap$ $\left\{x_{1} \geq \frac{3}{4} d_{\star}\right\}$,

$$
Z_{\varepsilon}^{\tau} \geq G-\eta
$$

This way,

$$
x_{1} \geq d_{\star} / 2 \Rightarrow Z_{\varepsilon}^{\tau}>\mathcal{L}\left(x_{1}\right)=\widetilde{\alpha}\left(x_{1}-d_{\star}\right)+\bar{B}(\eta, \mathcal{T}, \tau)
$$

where $\bar{B}(\eta, \mathcal{T}, \tau)=\widetilde{\alpha}\left(c_{1} \eta-\mathcal{T}-\tau\right)-\eta \rightarrow 0$ as $\eta, \tau \rightarrow 0$.

Since, $\mathcal{L}\left(d_{\star} / 2\right)>-\widetilde{\alpha} / 4>\kappa_{2} \eta$ for $\eta, \tau$ small enough, and $\frac{d}{d x_{1}} \mathcal{L}\left(x_{1}\right)=\widetilde{\alpha}>\alpha$, we conclude that

$$
x_{1} \geq d_{\star} / 2 \Rightarrow Z_{\varepsilon}^{\tau} \geq \mathcal{L}>v+\eta>v_{\varepsilon}
$$

and clearly,

$$
x_{1} \leq d_{\star} / 2 \Rightarrow Z_{\varepsilon}^{\tau}-v_{\varepsilon}>C \varepsilon-C_{\eta} \varepsilon^{3}>0
$$

This way, by the translation invariance of $\left(S E_{\varepsilon}\right)$, Remark (5.5), $Z_{\varepsilon}^{\tau}$ is a supersolution of $\left(S E_{\varepsilon}\right)$ in $Q_{00}$. Finally, for $\eta, \tau, \varepsilon$ small enough,

$$
\begin{gathered}
Z_{\varepsilon}^{\tau} \geq v_{\varepsilon} \text { in } Q_{00} \\
Z_{\varepsilon}^{\tau}\left(x_{\varepsilon}^{\tau}\right)<v_{\varepsilon}\left(x_{\varepsilon}^{\tau}\right) \text { with } x_{\varepsilon}^{\tau} \in \operatorname{int}\left(Q_{00}\right)
\end{gathered}
$$

which contradicts the fact that $v_{\varepsilon}$ is the least supersolution of $\left(S E_{\varepsilon}\right)$. This way, $H(\alpha) \geq M$ and the Theorem is proven.

\section{Limit Free Boundary Problem}

In this section, we prove that the limit of the least viscosity, $u_{0}$ provided by Theorem (4.1) is a solution in the Caffarelli's viscosity sense as well as in the pointwise sense $\left(\mathcal{H}^{N-1}\right.$ a.e. $)$ to the free boundary problem

$$
\begin{gathered}
\Delta u=0 \text { in } \Omega \backslash \partial\{u>0\} \\
H_{\nu}\left(u_{\nu}^{+}\right)-H_{\nu}\left(u_{\nu}^{-}\right)=M \text { on } \Omega \cap \partial\{u>0\}
\end{gathered}
$$


where $u^{+}=\max (u, 0), u^{-}=\max (-u, 0), \nu$ is the inward unit normal to the free boundary $F(u)=\Omega \cap \partial\{u>0\}$ and

$$
H_{\nu}(t)=\int_{0}^{t} \frac{s}{F(s \nu)} d s
$$

This notion of weak solution was introduced by Luis A. Caffarelli in the classical papers [C2, C3]. Now, we provide these definitions to our problem.

Definition 9.1. Let $\Omega$ be a domain in $\mathbb{R}^{N}$ and $u \in C^{0}(\Omega)$. Then, $u$ is called a viscosity supersolution to $(\mathrm{FBP})$ if

i) $\Delta u \leq 0$ in $\Omega^{+}=\Omega \cap\{u>0\}$

ii) $\Delta u \leq 0$ in $\Omega^{-}=\left(\Omega \backslash \Omega^{+}\right)^{\circ}$

iii) Along $F(u), u$ satisfies

$$
H_{\nu}\left(u_{\nu}^{+}\right)-H_{\nu}\left(u_{\nu}^{-}\right) \leq M
$$

in the following sense:

If $x_{0} \in F(u)$ is a regular point from the nonnegative side(i.e, there exists $B_{r}(y) \subset \Omega^{+}$with $\left.x_{0} \in \partial B_{r}\left(x_{0}\right)\right)$ and

$$
u^{+}(x) \geq \alpha\left\langle x-x_{0}, \nu\right\rangle^{+}+o\left(\left|x-x_{0}\right|\right) \text { in } B_{r}\left(x_{0}\right), \quad(\alpha>0)
$$

and

$$
u^{-}(x) \geq \beta\left\langle x-x_{0}, \nu\right\rangle^{-}+o\left(\left|x-x_{0}\right|\right) \text { in } B_{r}\left(x_{0}\right)^{C}, \quad(\beta \geq 0)
$$

with equality along every nontangential domain in both cases, then

$$
H_{\nu}(\alpha)-H_{\nu}(\beta) \leq M
$$

Analogously, we have

Definition 9.2. Let $\Omega$ be a domain in $\mathbb{R}^{N}$ and $u \in C^{0}(\Omega)$. Then, $u$ is called a viscosity subsolution to (FBP) if

i) $\Delta u \geq 0$ in $\Omega^{+}=\Omega \cap\{u>0\}$

ii) $\Delta u \geq 0$ in $\Omega^{-}=\left(\Omega \backslash \Omega^{+}\right)^{\circ}$ 
iii) Along $F(u), u$ satisfies

$$
H_{\nu}\left(u_{\nu}^{+}\right)-H_{\nu}\left(u_{\nu}^{-}\right) \geq M
$$

in the following sense:

If $x_{0} \in F(u)$ is a regular point from the nonpositive side (i.e, there exists $B_{r}(y) \subset \Omega^{-}$with $\left.x_{0} \in \partial B_{r}\left(x_{0}\right)\right)$ and

$$
u^{-}(x) \geq \beta\left\langle x-x_{0}, \nu\right\rangle^{-}+o\left(\left|x-x_{0}\right|\right) \text { in } B_{r}\left(x_{0}\right), \quad(\beta \geq 0)
$$

and

$$
u^{+}(x) \geq \alpha\left\langle x-x_{0}, \nu\right\rangle^{+}+o\left(\left|x-x_{0}\right|\right) \text { in } B_{r}\left(x_{0}\right)^{C}, \quad(\alpha \geq 0)
$$

with equality along every nontangential domain in both cases, then

$$
H_{\nu}(\alpha)-H_{\nu}(\beta) \geq M
$$

Remark 9.3. There are equivalent definitions for supersolutions and subsolutions to (FBP) above. We mention an equivalent one for supersolutions that will be used in the next results. For this and further details, see [CS], chapter 2 .

Equivalentely, $u \in C^{0}(\Omega)$ is a supersolution of (FBP) if conditions $i$ ), $i i$ ) of defintion (9.1) are satisfied and if $x_{0}$ is regular point from the nonnegative side with tangent ball $B$

$$
u^{+}(x) \geq \alpha\left\langle x-x_{0}, \nu\right\rangle^{+}+o\left(\left|x-x_{0}\right|\right) \text { in } B, \quad(\alpha \geq 0)
$$

then,

$$
u^{-}(x) \geq \beta\left\langle x-x_{0}, \nu\right\rangle^{-}+o\left(\left|x-x_{0}\right|\right) \text { in } B^{C}, \quad(\beta \geq 0)
$$

For any $\beta$ such that

$$
H_{\nu}(\alpha)-H_{\nu}(\beta)>M
$$

Now, we move towards the proof of the major results in this section. In what follows, $u_{0}$ will always denote the limit of the least supersolutions given by Theorem 4.1. 
Proposition 9.4. $u_{0}$ is a viscosity subsolution to (FBP)

Proof. Clearly, conditions $i$ ), $i i$ ) of definiton (9.1) are satisfied. Now, let us suppose that $x_{0} \in F\left(u_{0}\right)$ is a regular point from the nonpositive side with tangent ball $B$. We can assume without lost of generality that $x_{0}=0$ and $\nu=e_{1}$. This way, by linear behavior at regular boundary points, Lemma (11.7) in [CS, there exist $\alpha \geq 0$ and $\beta>0$

$$
u_{0}^{+}(x)=\alpha x_{1}^{+}+o(|x|) \text { in } B^{C}
$$

and

$$
u_{0}^{-}(x)=\beta x_{1}^{-}+o(|x|) \text { in } B .
$$

Since $u_{0}^{+}$is nodegenerate, by Theorem 4.1 e) or more specifically, since 4.1 holds, we conclude that $\alpha>0$ and thus, $B$ is tangent to $F\left(u_{0}\right)$. This way, $u_{0}$ admits full asymptotic development, i.e,

$$
u_{0}(x)=\alpha x_{1}^{+}-\beta x_{1}^{-}+o(|x|)
$$

Taking now any sequence $\lambda_{n} \rightarrow 0$ and using the blow-up sequence $\left(u_{\varepsilon_{k}^{\prime}}\right)_{\lambda_{n}}$ given in proposition (5.2), we conclude that there exists a subsequence that we still denote by $\varepsilon_{k}^{\prime}$ such that $\left(u_{\varepsilon_{k}^{\prime}}\right)_{\lambda_{k}} \rightarrow \alpha x_{1}^{+}-\beta x_{1}^{-}$uniformly in compact subsets of $\mathbb{R}^{N}$. Since by remark (5.5) the equation $\left(S E_{\varepsilon}\right)$ and the least supersolution property are preserved under blow-up process, by Theorem 8.1, we conclude that

$$
H(\alpha)-H(\beta)=M
$$

where $H=H_{e_{1}}$.

Proposition 9.5. $u_{0}$ is a supersolution to (FBP).

Proof. As we already observed, $u_{0}$ satisfies conditions $i$ ), ii) of definition (9.1). We will show that the condition in the remark (9.3) holds. This way, let us assume that $B=B_{r}(y)$ be a touching ball from the nonnegative side at $x_{0}$ and let us assume that for some $\alpha \geq 0$,

$$
u_{0}^{+}(x) \geq \alpha\left\langle x-x_{0}\right\rangle^{+}+o\left(\left|x-x_{0}\right|\right) \text { in } B
$$


where $\nu$ given by the inward unit radial direction of the ball at $x_{0}$. If $H_{\nu}(\alpha) \leq M$ there is nothing to prove. Otherwise, if

$$
H_{\nu}(\alpha)>M
$$

let $\gamma \geq 0$ such that $H_{\nu}(\alpha)-H_{\nu}(\gamma)>M$ (we can find such $\gamma$, since $H_{\nu}$ is a bijection from $[0,+\infty]$ into itself). We will show that

$$
u_{0}^{-}(x) \geq \gamma\left\langle x-x_{0}, \nu\right\rangle^{-}+o\left(\left|x-x_{0}\right|\right) \text { in } B^{C}
$$

As usual, we assume without loss of generality that $\nu=e_{1}$ and $x_{0}=0$. We will prove the following

Claim: There exist $\bar{\alpha}, \bar{\gamma}>0$ such that

$$
u_{0}(x)=\bar{\alpha} x_{1}^{+}-\bar{\gamma} x_{1}^{-}+o(|x|) .
$$

Indeed, by the Lemma (4.1) in [LW],

$$
u_{0}^{-}(x)=\bar{\gamma} x_{1}^{-}+o(|x|) \text { in }\left\{x_{1}<0\right\}
$$

for some $\bar{\gamma} \geq 0$. Let us consider the blow-up sequence, i.e, for $\lambda>0$,

$$
\left(u_{0}\right)_{\lambda}(x)=\frac{1}{\lambda} u_{0}(\lambda x) .
$$

Since $u_{0}$ is locally Lipschitz continuous and $u_{0}(0)=0$ then, for every sequence, $\lambda_{n} \rightarrow 0$, there exists a subsequence, that we still denote by $\lambda_{n}$, such that $\left(u_{0}\right)_{\lambda_{n}} \rightarrow U_{0}$ uniformly in compact sets of $\mathbb{R}^{N}$, where $U_{0}$ is Lipschitz in $\mathbb{R}^{N}$. By 9.1 and 9.4 we know that

$$
U_{0}^{-}=\bar{\gamma} x_{1}^{-} \text {in } \mathbb{R}^{N}
$$

and

$$
U_{0}>0 \text { and harmonic in }\left\{x_{1}>0\right\}
$$

We have to analyze two cases:

Case I: $\bar{\gamma}>0$. 
In this case, $U_{0}<0$ in $\left\{x_{1}<0\right\}$. Therefore $U_{0}=0$ on the hyperplane $\left\{x_{1}=0\right\}$ and since it is Lipschitz continuous, we have

$$
U_{0}^{+}(x)=\bar{\alpha} x_{1}^{+} \text {in } \mathbb{R}^{N}
$$

for some $\bar{\alpha}>0$. This way, we conclude that

$$
U_{0}(x)=\bar{\alpha} x_{1}^{+}-\bar{\gamma} x_{1}^{-}, \quad \bar{\alpha}, \bar{\gamma}>0
$$

Case II: $\bar{\gamma}=0$.

In this case, $U_{0} \geq 0$ in $\mathbb{R}^{N}$. Since $U_{0}>0$ and harmonic in harmonic in $\left\{x_{1}>0\right\}$, then by Lemma A1 in [C3], there exist $\bar{\alpha}>0$ such that

$$
U_{0}(x)=\bar{\alpha} x_{1}^{+}+o(|x|) \text { in }\left\{x_{1}>0\right\} .
$$

Since $\bar{\alpha}>\alpha$, then $\left(H=H_{e_{1}}\right)$

$$
H(\bar{\alpha}) \geq H(\alpha)>M
$$

Let us consider, for $\lambda>0$, the blow-up sequence

$$
\left(U_{0}\right)_{\lambda}(x)=\frac{1}{\lambda} U_{0}(\lambda x)
$$

Since $U_{0}$ is Lipschitz continuous and $U_{0}(0)=0$, there exists a subsequence $\bar{\lambda}_{n} \rightarrow 0$, such that $\left(U_{0}\right)_{\overline{\lambda_{n}}} \rightarrow U_{00}$ uniformly on compact sets of $\mathbb{R}^{N}$, where $U_{00} \in \operatorname{Lip}\left(\mathbb{R}^{N}\right)$. By 9.6 ,

$$
U_{00}(x)=\bar{\alpha} x_{1}^{+} \text {in }\left\{x_{1}>0\right\} .
$$

Let us observe that, $U_{00} \geq 0$ in $\mathbb{R}^{N}$, it is harmonic in its positivity set $\left\{U_{00}>0\right\}$ and $u_{00}=0$ on the hyperplane $\left\{x_{1}=0\right\}$, again by Lemma $\mathrm{A} 1$ in C3. we have

$$
u_{00}(x)=\widetilde{\alpha} x_{1}^{-}+o(|x|) \text { in }\left\{x_{1}<0\right\},
$$

for some $\widetilde{\alpha} \geq 0$. Finally, if we consider once more for $\lambda>0$ the blow-up sequence

$$
\left(u_{00}\right)_{\lambda}=\frac{1}{\lambda} u_{00}(\lambda x)
$$


As before, there is still a subsequence $\widetilde{\lambda}_{n} \rightarrow 0$ and $U_{000} \in \operatorname{Lip}\left(\mathbb{R}^{N}\right)$, such that $\left(U_{00}\right)_{\widetilde{\lambda_{n}}} \rightarrow U_{000}$ uniformly on compact subsets of $\mathbb{R}^{N}$. From the computations above, we conclude

$$
U_{000}(x)=\bar{\alpha} x_{1}^{+}+\widetilde{\alpha} x_{1}^{-}, \quad \bar{\alpha}>0, \widetilde{\alpha} \geq 0 .
$$

Applying proposition (5.2) and recalling that least supersolutions are preserved under blow-ups, we can see that there exists a sequence $\delta_{n} \rightarrow 0$ and least supersolutions $u_{\delta_{n}}$ to $\left(S E_{\delta_{n}}\right)$ such that

$$
u_{\delta_{n}} \rightarrow U_{0}
$$

uniformly on compact sets of $\mathbb{R}^{N}$. Applying the same poposition twice, we see that there exist a sequence $\widetilde{\delta_{n}} \rightarrow 0$ and solutions $u_{\widetilde{\delta_{n}}}$ to $\left(S E_{\widetilde{\delta}_{n}}\right)$ such that $u_{\widetilde{\delta_{n}}} \rightarrow U_{000}$ uniformly on compact sets of $\mathbb{R}^{N}$. By Theorem (8.1) and by 9.2

$$
H(\bar{\alpha}) \leq M<H(\alpha)
$$

which contradicts (9.7). Then, case II does not occur and 9.5 holds, proving the claim. This way, by 9.8 , we can apply again Theorem (8.1) to $U_{0}$ to conclude

$$
H_{e_{1}}(\bar{\alpha})-H_{e_{1}}(\bar{\gamma})=M
$$

By proposition (5.3), there exists a $\delta>0$ independent of the sequence $\lambda_{n}$ such that

$$
\overline{\alpha \gamma}=\delta
$$

So, $\bar{\alpha}$ and $\bar{\gamma}$ are determinded in a unique way and therefore, $U_{0}$ does not depend on the sequence $\lambda_{n}$. In particular,

$$
\left(u_{0}\right)_{\lambda} \rightarrow U_{0}
$$

uniformly in compact subsets of $\mathbb{R}^{N}$ (as $\lambda \rightarrow 0$ ). Thus,

$$
u_{0}(x)=\bar{\alpha} x_{1}^{+}-\bar{\gamma} x_{1}^{-}+o(|x|)
$$


In particular,

$$
u_{0}^{-}(x)=\bar{\gamma} x_{1}^{-}+o(|x|) \text { in } B^{C}
$$

By $(9.9)$, we obtain since $\bar{\alpha} \geq \alpha$

$$
H_{e_{1}}(\bar{\gamma})=H_{e_{1}}(\bar{\alpha})-M \geq H_{e_{1}}(\alpha)-M>H_{e_{1}}(\gamma)
$$

from which we conclude $\bar{\gamma}>\gamma$ and therefore by (9.11),

$$
u_{0}^{-}(x)>\gamma x_{1}^{-}+o(|x|) \text { in } B^{C}
$$

This finishes the proof.

We establish now, the pointwise result.

Theorem 9.6. For $\mathcal{H}^{n-1}$ a.e. $x_{0} \in F\left(u_{0}\right), u_{0}$ has the following asymptotic development

$$
u_{0}(x)=\alpha\left\langle x-x_{0}, \nu\right\rangle^{+}-\gamma\left\langle x-x_{0}, \nu\right\rangle^{-}+o\left(\left|x-x_{0}\right|\right)
$$

where

$$
H_{\nu}(\alpha)-H_{\nu}(\gamma)=M
$$

In particular, around such points, the free boundary $F\left(u_{0}\right)$ is flat in the sense of Theorem $2^{\prime}$ in $\mathrm{C} 2$.

Proof. Indeed, by Theorem 4.4), for $\mathcal{H}^{N-1}$ a.e. in $F\left(u_{0}\right)$ we have,

$$
u_{0}(x)=q_{u_{0}}^{+}\left(x_{0}\right)\left\langle x-x_{0}, \nu\right\rangle^{+}-q_{u_{0}}^{-}\left(x_{0}\right)\left\langle x-x_{0}, \nu\right\rangle^{-}+o\left(\left|x-x_{0}\right|\right)
$$

Considering now, the blow-up sequence, $\left(u_{0}\right)_{\lambda}(x)=\frac{1}{\lambda} u\left(x_{0}+\lambda x\right), \lambda>0$, we have

$$
\left(u_{0}\right)_{\lambda} \rightarrow q_{u_{0}}^{+}\left(x_{0}\right)\left\langle x-x_{0}, \nu\right\rangle^{+}-q_{u_{0}}^{-}\left(x_{0}\right)\left\langle x-x_{0}, \nu\right\rangle^{-}
$$

Since least supersolutions are preserved under blow-up process, as in the previous Theorem, by proposition (5.2) and Theorem (8.1), we conclude that

$$
H_{\nu}\left(q_{u_{0}}^{+}\left(x_{0}\right)\right)-H_{\nu}\left(q_{u_{0}}^{-}\left(x_{0}\right)\right)=M
$$

The flatness follows now by the arguments in [C3]. This finishes the proof. 
At last, we prove our last Theorem concerning about the regularity of the free boundary $F\left(u_{0}\right)$.

Theorem 9.7 (Free boundary regularity). Let $u_{0}$ be the limit of the least supersolutions given by Theorem (4.1). Then, the free boundary $F\left(u_{0}\right)=$ $\partial\left\{u_{0}>0\right\} \cap \Omega$ is a $C^{1, \gamma}$ surface in a neighborhood of $\mathcal{H}^{N-1}$ a.e. point $x_{0} \in$ $F\left(u_{0}\right)_{\text {red. }}$ In particular, $F\left(u_{0}\right)$ is a $C^{1, \gamma}$ surface in a neighborhood of $\mathcal{H}^{N-1}$ a.e. point in $F\left(u_{0}\right)$.

Proof. We already know that $u_{0}$ is a viscosity solution of $(\mathrm{FBP})$. In this case, $u_{0}$ satisfies

$$
u_{\nu}^{+}=G\left(u_{\nu}^{-}, \nu\right) \text { on } F(u)
$$

in the viscosity sense, where

$$
G(z, \nu)=H_{\nu}^{-1}\left(M+H_{\nu}(z)\right)
$$

Let us observe that $G$ depends on $\nu$ in a Lipschitz continuous fashion. Indeed, there is a constant $C>0$ such that $G(z, \nu) \geq C$. To see that, since $t^{2} / 2 F_{\max } \leq H_{\nu}(t) \leq t^{2} / 2 F_{\text {min }}$, for $t \geq 0$, we obtain

$$
\frac{G(z, \nu)^{2}}{2 F_{\min }} \geq H_{\nu}(G(z, \nu)) \geq M+H_{\nu}(z) \geq M
$$

Furthermore,

$$
\left|H_{\nu}(x)-H_{\nu}(y)\right| \geq \frac{\sigma}{F_{\min }}|x-y| \text { for } x, y \in[\sigma,+\infty) .
$$

This way, for $\nu_{1}, \nu_{2} \in \mathbb{S}^{N-1}$, by 9.13

$$
\mid H_{\nu_{1}}\left(G\left(z, \nu_{1}\right)-H_{\nu_{2}}\left(G\left(z, \nu_{2}\right)|=| H_{\nu_{1}}(z)-H_{\nu_{2}}(z) \mid\right.\right.
$$

therefore for $|z| \leq C_{0}$, there exists $\overline{C_{0}}=\overline{C_{0}}\left(C_{0}, \operatorname{Lip}(F)\right)$ such that

$$
\begin{gathered}
\frac{C}{F_{\text {min }}}\left|G\left(\nu_{1}, z\right)-G\left(\nu_{2}, z\right)\right| \leq \mid H_{\nu_{1}}\left(G\left(z, \nu_{1}\right)-H_{\nu_{2}}\left(G\left(z, \nu_{2}\right) \mid=\right.\right. \\
=\left|H_{\nu_{1}}(z)-H_{\nu_{2}}(z)\right| \leq \overline{C_{0}}\left|\nu_{1}-\nu_{2}\right|
\end{gathered}
$$

Moreover, by Theorem (4.1) $u_{0}$ is locally Lipschitz continuous and it has linear growth away from its free boundary $F\left(u_{0}\right)$. Also, since $F\left(u_{0}\right)_{\text {red }}$ has full 
$\mathcal{H}^{N-1}$ measure in $F\left(u_{0}\right), u_{0}$ is for $\mathcal{H}^{N-1}$ a.e. point on $F\left(u_{0}\right)$ a 2-plane solution. In particular, for any such point $x_{0}$, a suitable dilation

$$
\left(u_{0}\right)_{\tau}=\frac{u\left(\tau\left(x-x_{0}\right)\right)}{\tau}, \quad \tau \text { small enough }
$$

falls under conditions of Theorem 3 in [C2], concluding the proof.

\section{ACKWNOLEDGEMENTS}

The author is deeply grateful to his PhD advisor, Prof. Luis A. Caffarelli, by the deep and exciting mathematical discussions during the elaboration of this paper. The author is also grateful for all his support and encouragement.

\section{REFERENCES}

[ABR] Axler, Sheldon; Bourdon, Paul; Ramey, Wade Harmonic Functions Theory, Graduate texts in Mathematics No. 137, springer-Verlag New-York, 2001.

[AC] Alt, H. and Caffarelli, L. Existence and regularity for a minimum problem with free boundary. J. Reine Angew. Math. 325 (1981), 105-144.

[BCN] H. Berestycki, L. A. Caffarelli and L. Nirenberg, Uniform estimates for regularization of free boundary problems. Analysis and partial differential equations, 567-619, Lecture Notes in Pure and Appl. Math., 122, Dekker, New York, 1990.

[B] Blair, David inversion theory and conformal mapping Student mathematical library, No. 9 - American mathematical society - Provide, RI, 2000.

[C1] Caffarelli, Luis A. A Harnack inequality approach to the regularity of free boundaries. I. Lipschitz free boundaries are $C^{1, \alpha}$. Rev. Mat. Iberoamericana 3 (1987), no. 2, 139-162.

[C2] Caffarelli, Luis A. A Harnack inequality approach to the regularity of free boundaries. II. Flat free boundaries are Lipschitz. Comm. Pure Appl. Math. 42 (1989), no. 1, 55-78.

[C3] Caffarelli, Luis A. A Harnack inequality approach to the regularity of free boundaries. III. Existence theory, compactness, and dependence on X. Ann. Scuola Norm. Sup. Pisa Cl. Sci. (4) 15 (1988), no. 4, 583-602 (1989).

[C4] Caffarelli, Luis A. Uniform Lipschitz regularity of a singular pertubation problem. Differential and Integral Equations, Volume 8, Number 7, pp 1585- 1590 (1995) .

[CC] Caffarelli, Luis A., Cabre, Xavier, Fully nonlinear elliptic equations. American Mathematical Society Colloquium Publications, 43. American Mathematical Society, Providence, RI, 1995

[CLM1] Caffarelli, Luis A.; Lee, Ki-Ahm; Mellet, Antoine Singular limit and homogenization for flame propagation in periodic excitable media. Arch. Ration. Mech. Anal. 172 (2004), no. 2, 153-190.

[CLM2] Caffarelli, L. A.; Lee, K.-A.; Mellet, A. Homogenization and flame propagation in periodic excitable media: the asymptotic speed of propagation. Comm. Pure Appl. Math. 59 (2006), no. 4, 501-525 
[CJK] Caffarelli, Luis A., Jerison, David., Kenig, Carlos, Some new monotonicity Theorems with applications to free boundary problems, Annals of Mathematics, 155 (2002), 369 404.

[CLW1] Caffarelli, Luis A., Lederman, Claudia., Wolanski, Noemi, Uniform estimates and limits for a two phase parabolic singular pertubation problem. Indiana Univ. Math. J. 46(2), 1997, pp 453 - 490.

[CLW2] Caffarelli, Luis A., Lederman, Claudia., Wolanski, Noemi, Pointwise and viscosity solutions for the limit of a two phase parabolic singular perturbation problem. Indiana Univ. Math. J. 46(3), 1997, pp 719 - 740.

[CV] Caffarelli, Luis A., Vazques, Juan L. A free boundary problem for the heat equation arising in flame propagation, Trans. Amer. Math. Society, vol 347, 1995, 411 - 441.

[CS] Caffarelli, Luis A., Salsa, Sandro, Geometric approach to free boundary problems. Graduate Studies in Mathematics - Volume 68 - AMS - 2005

[CCKS] Caffarelli, Luis A., Crandall, M.G, Kocan, M and Swiech, A. On Viscosity solutions of fully nonlinear equations with measurable ingridients. Comm. Pure Appl. Math. 49 (1996), no. 4, 365-397

[CKSS] Crandal, M.G., Kocan, M., Soravia, P., Swiech, A., On the Equivalence of Various weak notions of solutions of elliptic PDES with measurable ingridients Pitman Res. Notes Math. Ser., 350, Longman, Harlow, 1996

[EG] Evans, L.C.; Gariepy, R.F. Measure Theory and Fine Properties of Functions. Studies in Advanced Mathematics (1992)

[FH] Lin, Fanghua; Han, Qing Elliptic Partial Differential Equations. Courant lectures notes. AMS, 1997.

[GT] D. Gilbarg and N. Trudinger, Elliptic Partial Differential Equations of second order. Springer-Verlag, New York, 1983.

[I] Ishii, Hitoshi Perron's method for Hamilton-Jacobi Equations. Duke Mathematical Journal, Vol. 55, No.2, 1987

[LL] Lieb, Elliot H.; Loss, Michael Analysis Graduate Studies in Mathematics, Vol. 14, American Mathematical Society (AMS), 1997

[LW] Lederman, C; Wolanski, N Viscosity solutions and regularity of the free boundary for the limit of an elliptic two phase singular pertubation problem. Ann. Scuola Norm. Sup. Pisa Cl. Sci. (4) 27 (1998), no. 2, 253-288 (1999)

[LWS] Littman, W; Weinberger, H.F; Stampacchia, G Regular points for elliptic equation with discontinuos coefficients. Ann. Scuola Norm. Sup. di Pisa (3) 17 (1963), 43-77

[MT] Moreira, Diego R, Teixeira, Eduardo V.O. A singular free boundary problem for elliptic equations in divergence form. To appear Calculus of Variations and Partial Differential Equations.

[Tr1] Trudinger, Neil On Regularity and existence of viscosity solutions of nonlinear second order, elliptic equations. Partial differential equations and the calculus of variations, Vol. II, 939-957, Progr. Nonlinear Differential Equations Appl., 2, Birkhuser Boston, Boston, MA, 1989 
[Tr2] Trudinger, Neil On Twice differentiability of viscosity solutions of nonlinear elliptic equations. Bull. Austral. Math. Soc. 39 (1989), no. 3, 443-447

[ZF] Ya.B.Zeldovich, D.A. Frank-Kamenestki, The theory of thermal propagation of flames. Zh. Fiz. Khim. 12, (1938), 100-105 (in Russian); English translation in " Collected Works of Ya.B. Zeldovich", vol 1, Princeton Univ. Press, 1992.

Department of Mathematics, University of Texas at Austin, RLM 12.128, Austin, Texas 78712-1082.

E-mail address: dmoreira@math.utexas.edu 